\title{
1 Host regulator PARP1 contributes to sex differences and immune responses in a mouse model of
}

\section{2 tuberculosis}

3 Stefanie Krug ${ }^{1,2}$, Alvaro A. Ordonez ${ }^{1,3,4}$, Mariah Klunk ${ }^{1,3}$, Bong Gu Kang ${ }^{5,6}$, Sanjay K. Jain ${ }^{1,3,4}$, Ted M.

4 Dawson ${ }^{5,6,8,9}$, Valina L. Dawson ${ }^{5,6,7,8}$, William R. Bishai ${ }^{1,2 *}$

\section{Affiliations:}

$6{ }^{1}$ Center for Tuberculosis Research, Johns Hopkins University School of Medicine, Baltimore, MD, USA.

$7 \quad{ }^{2}$ Department of Medicine, Johns Hopkins University School of Medicine, Baltimore, MD, USA.

$8{ }^{3}$ Center for Infection and Inflammation Imaging Research, Johns Hopkins University School of Medicine,

9 Baltimore, MD, USA.

$10{ }^{4}$ Department of Pediatrics, Johns Hopkins University School of Medicine, Baltimore, MD, USA.

$11{ }^{5}$ Neuroregeneration and Stem Cell Programs, Institute for Cell Engineering, Johns Hopkins University

12 School of Medicine, Baltimore, MD, USA.

$13{ }^{6}$ Department of Neurology, Johns Hopkins University School of Medicine, Baltimore, MD, USA.

$14{ }^{7}$ Department of Physiology, Johns Hopkins University School of Medicine, Baltimore, MD, USA.

15 Solomon H. Snyder Department of Neuroscience, Johns Hopkins University School of Medicine,

16 Baltimore, MD, USA.

$17{ }^{9}$ Department of Pharmacology and Molecular Sciences, Johns Hopkins University School of Medicine,

18 Baltimore, MD, USA.

*Corresponding author: William R. Bishai, wbishai1@jhmi.edu, Phone number: 1-410-955-3507

Keywords: Mycobacterium tuberculosis; PARP1; sex differences; inflammation 


\section{Abstract:}

22 Tuberculosis (TB) is a devastating infectious disease responsible for nearly 2 million deaths annually that

23 has a poorly understood male bias. Elucidating the basis of this male bias may enable precision medicine

24 interventions for TB treatment and prevention. Here, we identify the master regulator Poly(ADP-ribose)

25 Polymerase 1 (PARP1) as a driver of TB sex differences. We found that infection with $M$. tuberculosis

$26(M . t b)$ triggers robust PARP activation in mouse lungs, suggesting that PARP1 activation is a

27 fundamental host response to TB. Remarkably, PARP1 deletion abolished known sex differences in TB

28 cytokine responses and blunted the early induction of TNF $\alpha$, IL-1ß, IFN $\gamma$, MCP-1, and IL-6, particularly in

29 male mice. In contrast, PARP1 was required for IL-10 induction in male or female mice. PARP1 deletion

30 was protective against TB in female mice, resulting in significantly prolonged survival and reduced

31 bacterial burden, but impaired TB containment in male mice. Our findings indicate that PARP1

32 contributes to TB sex differences via sexually divergent immune regulation and uniquely enhances early

33 proinflammatory responses in males that prove beneficial for TB containment. 
Introduction:

Tuberculosis (TB) is a devastating global health threat responsible for over 1.5 million deaths every year (1). TB has an appreciated male bias, with nearly twice as many adult men developing active TB as women (2-4). Moreover, TB mortality, lesion severity, cavitation rates and treatment responses tend to be worse in men than in women (5-8). A male bias in disease severity and lethality has also been demonstrated in mice infected with Mycobacterium tuberculosis (M. tb) and non-tuberculous mycobacteria, including M. marinum and M. avium (9-12). While these patterns appear to be driven primarily by biological factors, surprisingly little is known about genetic and hormonal factors which underlie this male bias $(4,13)$.

The eukaryotic master regulator Poly(ADP-ribose) Polymerase-1 (PARP1) has emerged as a critical cofactor of NF-KB activation and TNF $\alpha$ signaling but its role in TB has not yet been studied (1418). PARP1 is a nuclear enzyme that regulates fundamental cellular functions by modifying nuclear proteins, including itself, and sites of DNA damage post-translationally with poly(ADP-ribose) polymers (PAR) in a process termed PARylation $(15,19-22)$. PARylation is a potent, multi-faceted stress response element that can trigger increased proinflammatory gene expression, DNA repair, metabolic changes, and cell death $(15,23,24)$. PARP1 also controls numerous immune functions, including immune cell

51 activation, differentiation and recruitment; NF-KB signaling; and the production of TNF $\alpha$, IL-6, IL-1ß,

52 IFN $\gamma$, MCP-1 and nitric oxide (NO) $(14,23,25)$. Furthermore, PARP1 amplifies and sustains inflammation by inducing proinflammatory mediators and oxidative stress conditions, which in turn continue to

54 stimulate PARP1 activity in a positive-feedback loop $(23,25)$. Consequently, PARP1 has been connected

55 to inflammatory disorders, including endotoxic shock, sepsis, asthma, COPD and ARDS, and PARP inhibition reduces inflammation and disease severity in numerous inflammatory conditions (23-26).

57 Since PARP1 regulates key processes that are important for TB containment as well as TB

58 immunopathology, we hypothesized that PARP1 might be a core component of the host response to TB. 
PARP1 is also a regulator of biological sex differences, including in stress resistance,

60 inflammation and aging (27-29). PARP1 is involved in silencing the inactive $X$ chromosome, which

61 contains genes involved in pathogen recognition and cytokine signaling, sex hormone signaling, and

62 sexually divergent cell death mechanisms (27, 30-34). Consequently, sexually divergent effects of PARP1

63 deletion or inhibition have been reported in cell death, brain injury, inflammation, nephrotoxicity,

64 carcinogenesis and aging $(29,30,32,34-38)$. Therefore, PARP1 might have distinct functions in the host

65 response to TB in men and women and similarly contribute to sexually divergent immune regulation in

66 TB infection.

We therefore investigated whether PARP1 may regulate the TB host response, inflammation and sex effects. Here, we demonstrate that PARP1 is activated during $M$. tb infection in mice and serves

69 as a driver of TB inflammation and lung damage. Interestingly, PARP1 deficiency in knockout mice

70 reduced elevated proinflammatory responses in male WT mice to female levels but was only protective

71 in female mice. Our results indicate that PARP1 is an important driver of TB sex differences; in males it

72 uniquely enhances early proinflammatory cytokine production resulting in improved male TB

73 containment.

74 Results:

75 TB infection activates PARP1 in mouse lungs

PARP1 is a host enzyme that regulates cellular functions by post-translationally modifying its

77 targets with poly(ADP-ribose) polymers (PAR) (15). Since these polymers are rapidly degraded, PARP1

78 activity can be assessed by quantifying PAR levels, which appear as high molecular weight smears on a

79 PAR immunoblot with a pronounced focus around $116 \mathrm{kDa}$ resulting from PARP1 automodification (24,

80 39). To determine if PARP1 is activated during TB infection, we first evaluated PAR levels in the lungs of

81 age-matched uninfected or $M$. tb-infected C3HeB/FeJ mice (Fig. 1a-b). While PAR levels were typically 
82 low in uninfected lungs, we detected robust PAR signals in infected lungs that increased over the course

83 of infection, with levels 20 - and 80 -fold higher than in uninfected lungs $(p<0.02)$ at 60 and 120 days post

84 infection (dpi), respectively (Fig. 1a-b).

We next assessed the PAR response to infection in three additional mouse strains routinely used to model TB: C57BL/6, 129S2, and BALB/c mice (Fig. 1c-e). All strains contained bacterial numbers to

87 around $6 \log _{10}$ CFUs in the lung following low-dose aerosol infection, with no differences in bacterial burden and modest pathology after 7 weeks of infection (not shown). Despite variable baseline PARP1 activity in uninfected lungs, $M . t b$ infection consistently induced PAR formation in all three strains, suggesting that PARP1 activation might be a conserved host response to TB. Importantly, treatment with

91 the PARP inhibitor talazoparib (Tp) reduced PAR formation in $M . t b$-infected lungs in a dose-dependent 92 manner, indicating that $M$. tb-induced PARP1 activation can be reversed pharmacologically (Fig. 2).

93 Therefore, PARP1 activation appears to be a core component of the host response to $M$. $t b$. infection and may play a critical host regulatory role in TB.

PARP1 promotes proinflammatory immune responses in male but not in female mice

97 (PARP1 ${ }^{-1}$ ) and control 129S1/SvImJ (WT) mice with M. tb H37Rv via the aerosol route and evaluated

98 immune responses, disease progression, inflammation and survival. In 129S-Parp1 ${ }^{\text {tm1Zqw }} / \mathrm{J}$ mice, exon 2

99 of PARP1 is disrupted, and no enzymatic PARP1 activity is detectable in homozygous mutants (40).

100 These PARP1-deficient mice are viable, fertile, and display no physical or behavioral abnormalities (28).

101 We routinely verified the genotype of PARP1 $1^{--}$mice by PCR and confirmed the absence of PARP1 protein 102 by Western blot (Supplementary Fig. S1). 
105

106

107

108

109

110

111

112

113

114

115

infection $(14,23,41)$. After confirming that uninfected WT and PARP1 ${ }^{--}$mice produce comparable lung levels of cytokines and chemokines (Supplementary Fig. S2), we next compared the induction of these factors in male and female WT and PARP1 ${ }^{--}$mice over the course of infection. Dibbern et al. previously reported that male C57BL/6 mice produce higher levels of proinflammatory cytokines one month after M. tb infection than female mice (9). Consistent with their findings, we observed markedly higher induction of the proinflammatory cytokines and chemokines, TNF $\alpha$, IFN $\gamma$, IL-6, IL-1 $\beta$ and MCP-1, in 129S1 WT males compared to WT females by 28 dpi (Fig. 3a). Remarkably, PARP1 deletion abolished these sex differences and reduced the levels of proinflammatory cytokines in male mice at $28 \mathrm{dpi}$ to female levels (Fig. 3b). In contrast, lung levels of IL-2, IL-12, IL-17 and MIP-1 $\alpha$ were largely comparable between uninfected and infected mice and appeared to be independent of PARP1 or sex

(Supplementary Fig. S3).

Male WT mice displayed enhanced proinflammatory TB responses compared to PARP1 ${ }^{-}$males early in infection (28 dpi) but not in the chronic phase (74 dpi) (Fig. 4a; Supplementary Fig. S3c-d). Interestingly, PARP1 deletion only impaired proinflammatory responses in male but not in female mice (Fig. 4a-b), suggesting that PARP1 enhances the production of proinflammatory mediators in TB in a sexand time-dependent manner. In contrast, induction of IL-10, a regulatory cytokine that is modestly induced in TB and associated with improved containment and reduced tissue damage, was absent in both male and female PARP1 ${ }^{--}$mice, indicating that PARP1 is required for IL-10 production in a sexindependent manner (Fig. 4c-d) (42). Together, these data show that PARP1 deletion dampened early proinflammatory immune responses, including TNF $\alpha$, IL-1ß and IFN $\gamma$ production specifically in male mice, but eliminated IL-10 responses in both male and female mice.

\section{PARP1 deletion is protective and improves TB containment in female mice}

Since PARP1 enhances proinflammatory responses important for TB containment in male but not in female mice, we hypothesized that PARP1 ${ }^{-}$mice, in particular males, would be more susceptible 
129

130

131

132

133

134

135

136

to TB than WT mice. We therefore compared survival and disease progression in WT and PARP1 ${ }^{-}$mice following aerosol infection with M. tb H37Rv. Unexpectedly, PARP1 ${ }^{-}$mice appeared to be somewhat protected and survived moderate infection longer than WT mice (median survival: 109.5 in PARP1\% vs. 93 days in WT mice; not significant) (Table 1; Fig. 5). Surprisingly, we discovered that this protective effect of PARP1 deletion was due to the profound resistance of PARP1 ${ }^{--}$females, who survived infection significantly longer than WT females (134 vs. 83.5 days, respectively; $p=0.0031$; hazard ratio 5.225) (Fig. 5b; Table 1). In contrast, survival did not differ between WT and PARP1\% males (Fig. 5c; Table 1). These data indicate that PARP1 deletion has sexually divergent effects and prolongs female survival in TB.

In line with these observations, PARP1 ${ }^{-/-}$females contained $M$. tb. proliferation more efficiently than PARP ${ }^{-/-}$males and never exceeded a lung bacterial burden of $6.7 \pm 0.3 \log _{10}$ CFUs (Fig. 6a, left). By comparison, the lung bacterial burdens peaked at $7.3 \pm 0.3 \log _{10}$ CFUs in WT females ( $28 \mathrm{dpi}$ ), at $7.2 \pm$ $0.3 \log _{10}$ CFUs in WT males (42 dpi) and at $7.3 \pm 0.5 \log _{10}$ CFUs in PARP1 ${ }^{--}$males (28 dpi). Similarly, the bacterial burden in the spleen tended to be lowest in PARP1 ${ }^{-/}$females and highest in PARP1 $1^{--}$males (Fig. 6a, right). We consistently observed these same patterns in three independent experiments with similar infection parameters and day 1 lung implantations ranging from $1.66-1.86 \log _{10}$ CFUs. The combined analysis of these studies confirmed that PARP1 ${ }^{--}$females have significantly fewer lung bacteria than WT females one month after infection $(p<0.05)$, resulting in an overall lower bacterial burden in PARP1 ${ }^{--}$mice (Fig. 6b). These results suggest that PARP1 deletion is beneficial in female mice and increases TB survival by enhancing the control of bacterial replication.

\section{PARP1 deletion impairs TB control and exacerbates inflammation in male mice}

Unlike females, $\mathrm{PARP}^{-/-}$males contained early M. $t b$. infection similar to WT mice but developed higher lung and spleen bacterial burdens than any other group after $2.5-3$ months of infection (Fig. 6). As a result, PARP1 ${ }^{-}$mice (males and females combined) had significantly more lung bacteria than WT mice by three months post infection $(p<0.05)$, driven by the high bacterial burden in 
$\mathrm{PARP}^{-/}$males $(p<0.01)$ (Fig. 6c). Even though TB survival did not differ significantly in males, the hazard ratios indicate that the risk of death was nearly $30 \%$ lower in WT males than in PARP1 ${ }^{--}$males following infection (Table 1). Therefore, in contrast to females, PARP1 deletion is detrimental in males and antagonizes bacterial containment. appeared notably enlarged after 2-3 months of infection in all experiments (Fig. 7a; Supplementary Fig. S4). In fact, after three months of infection, the lungs of PARP1 ${ }^{-/}$males were approximately 1.5 -times heavier than those of any other group, even though PARP1 ${ }^{-/}$males weighed less than WT males and the lung weights of age-matched uninfected mice were comparable across all groups (Fig. 7b-c). Histopathologic analysis revealed that PARP1 ${ }^{-/}$males had significantly more lung inflammation and

163 consolidation than mice of any other group ( $p<0.05)$ (Fig. 7d-f; Supplementary Fig. S5). We also assessed lung inflammation in 3 additional mice by ${ }^{18}$ F-FDG PET and by CT but did not observe significant differences between groups (Supplementary Fig. S6). Together, these data suggest that PARP1 deletion impairs immune clearance of $M . t b$ and increases lung inflammation during chronic TB in males but not 167 in females.

171 Supplementary Fig. S3c) (9). Despite these sex differences in immune responses, TB progression and

172 inflammation did not differ between male and female 129S1 WT mice (Supplementary Fig. S7),

173 suggesting that there might be fundamental differences in the mechanism of TB containment in males

174 and in females. PARP1 deletion eliminated the sex difference in immune responses (Fig. $\mathbf{3 b}$ ) but

175 impaired TB containment in males and improved it in females (Fig. 2a; Fig. 8). Therefore, the PARP1- 
dependent enhancement of early male cytokine responses may be a compensatory mechanism required to achieve comparable TB containment in males and females.

\section{Discussion:}

TB has a male bias, associated primarily with biological rather than behavioral factors, but the underlying mechanisms are largely unknown (4). Here, we identified the master regulator PARP1 as a nuclear factor that contributes to sexually divergent TB immune responses and disease susceptibility. Despite comparable rates of infection, men progress to active TB not only more frequently but also with higher severity and mortality than women (3-7). A male bias in TB severity and mortality has also been reported in C57BL/6 and BALB/c mice; curiously, this was associated with increased early proinflammatory immune responses in male C57BL/6 mice whereas in BALB/c mice, the inverse was observed $(9,10)$. While we saw no clear sex differences in TB progression in 129S1/SvImJ mice, as has been described in C57BL/6 mice, we observed markedly enhanced production of proinflammatory mediators after one month in infected males that depended on the presence of functional PARP1. Interestingly, a similar role for sexually divergent immune regulation has been described for PARP1 in a mouse model of LPS-induced systemic inflammation: while females are highly resistant to LPS, males produce high levels of TNF $\alpha$ and die rapidly after LPS exposure, but PARP1 deletion or inhibition reduces TNF $\alpha$ levels and death in males to female levels (29). Therefore, PARP1 might also drive TB sex differences via the differential regulation of male and female immune responses, particularly during early infection.

Since high levels of inflammatory cytokines can contribute to bacterial containment but also promote immunopathology, exaggerated immune responses might thus be associated with worse TB outcomes. However, our findings indicate that robust proinflammatory responses early in infection are critical for long-term TB containment in males but not in females. Lack of PARP1 in male mice lowered 
cytokine responses to female levels but was associated with impaired bacterial control and paradoxically exacerbated inflammation during the chronic phase of infection. Since PARP1 drives proinflammatory responses in males, we expected WT males to have higher levels of tissue damage and lung inflammation and were surprised to observe more inflammation in PARP1 ${ }^{--}$males despite reduced proinflammatory responses. One explanation for these observations is that TB control in males might rely more heavily on cytokine-driven early bactericidal mechanisms than in females. Attenuation of this capacity in PARP1 ${ }^{--}$males might limit their ability to restrict bacterial replication, over time resulting in increased dissemination and lung damage. Therefore, males might compensate for fundamental sex differences in immune functions that are disadvantageous in TB by boosting early bactericidal mechanisms with the PARP1-dependent enhancement of proinflammatory mediators (Fig. 8). 
223

224

225

226

227

228

229

230

231

232

233

234

235

236

237

though IL-10 induction was PARP1-dependent in males and in females, the absence of IL-10 might impact TB progression differently in males and in females depending on the respective immune context. IL-10 can benefit both the pathogen, e.g., by suppressing innate and adaptive immune responses, and the host, e.g., by reducing tissue damage (44). In contrast to male mice, PARP1 deletion was profoundly protective in females, who exhibited significantly lower bacterial counts and prolonged survival than their WT counterparts. The only observed difference in immune responses between WT and PARP1 ${ }^{-}$ females was the lack of IL-10 induction in PARP1 ${ }^{-/}$mice (Fig. 4). Since IL-10 deletion or inhibition is associated with increased bacterial restriction and survival in mouse models of TB, lack of IL-10 could account for the improved survival and bacterial containment in PARP1 ${ }^{-/-}$females (42). The bacterial burden was most reduced In PARP1 ${ }^{-/}$females one month into infection, around the time when the initial period of unrestricted bacterial replication ends due to the onset of adaptive immunity (45). Therefore, the TB resistance of PARP1 $1^{--}$females might be the result of improved activation of adaptive immune responses, potentially because of absent immunosuppressive effects of IL-10. Importantly, since PARP1 deletion was profoundly protective in infected females, adjunctive PARP inhibition has the potential to improve TB clearance, particularly in women.

While our study revealed new insight into TB sex differences, further underlying mechanisms behind the male bias remain to be elucidated. The focus of our study was the comparison of disease progression and immune responses in WT and PARP1 ${ }^{-1}$ mice. However, it is entirely possible that the sexually dimorphic phenotype of PARP1 deletion in TB is only indirectly related to its function as an immune regulator. For example, PARP1 might drive TB sex differences via sexually dimorphic cell death paradigms. PARP1 regulates cell death signaling and acts as a switch between cell fates: PARP1 inhibits caspase activation and is reciprocally inactivated by caspases; PARP1 can induce caspase-independent, apoptotic as well as various forms of necrotic cell death; and PARP1 is implicated in RIPK-dependent and -independent forms of necroptosis $(38,46)$. In TB, apoptosis is host-protective by killing intracellular 
bacteria and stimulating adaptive immunity while necrosis is associated with bacterial dissemination and disease severity (44). Interestingly, cell death is sexually dimorphic and predominantly caspasemediated in females but caspase-independent, PARP1-mediated in males $(27,31,34,47)$. Accordingly, PARP1 deletion reduces cell death in males but increases caspase activity and cell death in females (27, 34, 47). Importantly, PARP inhibition reduces necrotic and increases apoptotic and live cell population, thereby shifting from a necrotic (unfavorable in TB) to an apoptotic (host-protective) milieu (28).

253 Therefore, TB sex differences might be the result of divergent cell death modalities that were

254 highlighted by PARP1 deletion. For example, by enhancing apoptosis in $M$. $t b$-infected females, PARP1

255 deficiency could potentially promote adaptive immune activation and accelerate TB containment, 256 resulting in lower bacterial burden in female mice around one month post infection that remain well 257 controlled throughout the chronic phase. Additional studies are needed to determine whether divergent 258 cell death paradigms contribute to TB sex differences in a PARP1-dependent or -independent manner. In 259 addition, our study did not assess the hormonal contribution toward the male bias in TB. PARP1 is indispensable for steroid sex hormone signaling, and sex hormones reciprocally activate PARP1 (32, 33,

$26138,48)$. TB infection studies utilizing castrated and ovariectomized mice and sex hormone

262 supplementation might therefore yield additional insight into the role of sex hormones to TB sex 263 differences.

In summary, we showed that PARP1 is robustly activated during $M . t b$. infection and contributes to immune responses and the control of infection in a sexually divergent manner. In contrast to LPS-

266 induced inflammation or infarct models, where PARP1 deficiency mainly benefits males, PARP1 deletion 267 in TB was protective in female but detrimental in male mice $(28,29)$. The impact of PARP1 deficiency in 268 TB was also less pronounced than in other proinflammatory conditions, such as endotoxic shock and LPS-induced lung injury $(17,49)$. These contrasting results likely reflect the disproportionate complexity 270 of host-pathogen interactions over the course of TB infection, not all of which are recapitulated in a 
271 mouse model. The fact that PARP1 activity in healthy human volunteers is significantly higher in men

272 than in women supports a sexually divergent role of PARP1 in humans $(28,32)$. Based on our findings,

273 we propose that in TB PARP1 is host-protective in males by enhancing early pro-inflammatory cytokine

274 responses, but antagonistic in females which except for IL-10 do not show major changes in PARP1-

275 dependent cytokine expression. Since PARP1 deletion was profoundly protective in infected female

276 mice, adjunctive PARP inhibition may have the potential to improve TB clearance, particularly in women.

277 In conclusion, PARP1 deletion revealed fundamental differences in the male and female response to TB

278 and might pave the way to a better understanding of TB sex differences. 
Methods:

281 Animals

282 Six-week-old female C57BL/6J (stock \#000664) and C3HeB/FeJ (stock \#658) mice were purchased from

283 The Jackson Laboratory, and female BALB/c (strain \#028) and 129S2/SvPasCrl (strain \#476) mice from

284 Charles River Laboratories. PARP1-deficient 129S-Parp1 ${ }^{\text {tm1Zqw } / J ~ m i c e ~(s t o c k ~ \# 002779, ~ J a c k s o n ~ L a b s) ~ w e r e ~}$

285 bred in-house. PARP1 disruption was routinely validated by PCR as described by the supplier (protocol

286 22839, version 2.3) using the common forward primer (5'-CATGTTCGATGGGAAAGTCCC-3'), a WT

287 reverse primer (5'-CCAGCGCAGCTCAGAGAAGCCA-3') and a mutant reverse primer (5'-

288 AGGTGAGATGACAGGAGATC-3'). Age-matched male and female recommended control 129S1/SvlmJ

289 mice (stock \#002448, Jackson Labs) were purchased for each experiment. All animal procedures were

290 approved by the Institutional Animal Care and Use Committee of the Johns Hopkins University School of

291 Medicine.

\section{Bacterial strains}

M. tuberculosis strains H37Rv and H37Rv $\Delta p n c A$ were obtained from the Johns Hopkins Center for

294 Tuberculosis Research, grown to an optical density at $600 \mathrm{~nm}$ of approximately 1.0 in Middlebrook 7H9

295 broth (Gibco) supplemented with $10 \%$ (v/v) oleic acid-albumin-dextrose-catalase (OADC; Difco), $0.5 \%$

296 (v/v) glycerol and $0.05 \%$ (v/v) Tween 80 (Sigma-Aldrich) and stored in $1 \mathrm{ml}$ aliquots at $-80^{\circ} \mathrm{C}$.

\section{Aerosol infections}

298 Mice were infected between 8 and 12 weeks of age via the aerosol route using the Glas-Col Inhalation

299 Exposure System (Terre Haute, IN). A new stock of $M$. tuberculosis was used for each infection and

300 diluted in sterile phosphate-buffered saline (PBS, pH 7.4) or 7H9 broth with OADC, glycerol, and Tween

30180 at empirically determined factors to achieve the desired inoculum. For high-dose infections (day 1

302 implantation $>2.5 \log _{10}$ CFUs), mycobacteria were thawed one week prior to infection and sub-cultured 
to achieve mid-log phase on the day of infection. For low-dose infections (day 1 implantation $<2.5 \log _{10}$

304 CFUs), a mycobacterial stock was thawed on the day of infection and used immediately. To reduce intergroup variability, mice from all comparative groups were infected together or evenly distributed between infection cycles (i.e., equal numbers of male and female PARP1 ${ }^{--}$and WT mice were infected in the same cycle). On the day after infection, 3-5 mice per cycle were sacrificed to determine the number of CFUs implanted into the lungs. The general appearance and body weight of mice were monitored at least weekly throughout all experiments. All infections, housing of infected mice and handling of infectious materials were carried out under biosafety level 3 containment in dedicated facilities. orogastric gavage once-daily for 5 consecutive days, in a total volume of $0.2 \mathrm{ml}$ per treatment. 
Aldrich). Plates were incubated at $37^{\circ} \mathrm{C}$ for $3-4$ weeks before colonies were counted. Colony numbers

327 were adjusted by the plating, dilution and dissection factors and log-transformed to estimate the total colony-forming units (CFUs) per organ (CFUs $=\log _{10}[($ number of colonies)*(5)*(A+B) / (A)] + [dilution factor]).

PARP1 activity was assessed by comparing poly(ADP-ribose) (PAR) levels in experimental and control

samples by immunoblot $(24,39)$. Lung sections were placed in chilled extraction buffer $(50 \mathrm{mM}$ glucose, 
specific for target-bound PAR, custom-designed at Bio-Rad AbD Serotec $\mathrm{GmbH}$ ) (39) with gentle agitation overnight at $4^{\circ} \mathrm{C}$. Following primary antibody incubation, the membrane was washed, incubated with HRP-conjugated goat anti-human IgG (Fab')2 (1:5,000 in 1\% nonfat dry milk-TBST; Abcam) for $1 \mathrm{~h}$ at RT and visualized with KwikQuant Ultra digital ECL substrate using the KwikQuant digital imager (Kindle Biosciences, LLC).

After visualizing PAR bands, the membrane was washed with TBS and stripped in a $200 \mathrm{mM}$ glycine solution ( $\mathrm{pH} 2.5$ ) for $1 \mathrm{~h}$ at RT. The membrane was washed with TBS and TBST, blocked in TBST containing $5 \%(\mathrm{w} / \mathrm{v})$ nonfat dry milk for $1 \mathrm{~h}$ at RT, washed with TBST, incubated with HRP-conjugated

357 mouse monoclonal anti-beta Actin antibody [AC-15] (1:50,000 in 5\% (w/v) BSA in TBST; Abcam) for 0.5-1

$358 \mathrm{~h}$ at RT and visualized as described for PAR detection. For PARP1 protein detection, membrane was

359 incubated with the primary HRP-conjugated (1:1,000 in 5\% nonfat dry milk; Cell Signaling Technology)

360 overnight at $4^{\circ} \mathrm{C}$ and secondary HRP-conjugated anti-rabbit IgG (1:2,000 in 5\% nonfat dry milk; Cell

361 Signaling Technology) for $1 \mathrm{~h}$ at RT. Digital images were converted to black-and-white using Photoshop,

362 and relative band intensities were quantified using ImageJ (version 1.52a) as described (50). PARP1

363 activity was defined as the intensity of high-molecular weight (72-250 kDa) PAR bands after

364 normalizing to $\beta$-actin and was expressed as fold change relative to uninfected or untreated control

365 samples on the same immunoblot.

\section{Cytokine and chemokine analysis}

367 Lung cytokines/chemokines (GMCSF, IFNY, IL-1ß, IL-2, IL-6, IL-10, IL-12(p70), IL-17/CTLA8, MCP-1/CCL2, 368 MIP-1 $\alpha /$ CCL3, TNF $\alpha$ ) were analyzed by Luminex multiplex bead assay on a Bio-Plex 200 platform (Bio369 Rad) with a mouse cytokine/chemokine magnetic bead panel (MCYTOMAG-70K, lot \# 3224392;

370 Millipore) according to the manufacturer's instructions. Lung tissue was disaggregated in a mini-

371 beadbeater (Biospec Products) at 4,800 RPM with $1.0 \mathrm{~mm}$ zirconia beads ( $5.5 \mathrm{~g} / \mathrm{cc}$; Biospec Products) in 
372 sterile PBS, incubated on ice for $5 \mathrm{~min}$ and centrifuged at $12,000 \mathrm{xg}$ for $10 \mathrm{~min}$. Supernatants were filter-

373 sterilized through $0.22 \mu \mathrm{m}$ cellulose acetate Spin-X centrifuge tube filters (Costar) and stored at $-80^{\circ} \mathrm{C}$.

374 Protein concentrations were determined by CB-X protein assay (G-Biosciences) according to the

375 manufacturer's instructions, and samples were diluted to $1 \mathrm{mg} / \mathrm{ml}$ for analysis. Results are presented as

376 concentrations $(\mathrm{pg} / \mathrm{ml})$ or fold change relative to uninfected lungs from the same group.

378 For histology, intact lungs were fixed by immersion in 10\% neutral-buffered formalin for 48 hours,

379 paraffin-embedded, sectioned and stained with hematoxylin and eosin (H\&E), Masson's trichrome or

380 Ziehl-Neelsen stain for acid-fast bacilli. Slides were digitally scanned at 40x on an Aperio AT turbo

381 scanner console version 102.0.7.5 (Leica Biosystems). Image files were transferred using Concentriq for

382 Research version 2.2.4 (Proscia Inc), randomized and scored blinded to experimental grouping using

383 Aperio ImageScope (Leica Biosystems). Regions of interest (ROI) were manually selected at 20x

384 magnification. Inflammatory regions were defined by leukocyte disruption of alveolar architecture. Lung 385 involvement was calculated as a percentage of the total lung area.

386 For ${ }^{18}$ F-FDG PET/CT, live animals were imaged at 84 days post-infection inside in-house developed,

387 sealed biocontainment devices compliant with BSL-3 isolation (51). Mice were fasted overnight, and ${ }^{18} \mathrm{~F}-$

388 FDG (5.31 $\pm 0.25 \mathrm{MBq})$ was administered intravenously via the tail vein. A 15-minute PET acquisition and

389 CT were performed using the nanoScan PET/CT (Mediso, Washington, DC) 45 min after tracer injection.

390 Volumes of interest (VOIs) were manually selected using CT as a guide and applied to the PET dataset

391 using VivoQuant 2020 (Invicro, Boston, MA). Mean lung ${ }^{18}$ F-FDG PET activity was calculated for each

392 mouse as the average activity of all VOIs normalized by injected dose and body weight and is expressed 393 as standard uptake values (SUV). 
397 Statistical analyses were performed using Prism version 5.01 for Windows (GraphPad) or Microsoft Excel

398 (Microsoft Office Professional Plus 2019). Statistical tests used are indicated in the figure legends.

399 Differences between two group means were assessed by unpaired, two-tailed $t$-test, with Welch's

400 correction for unequal variance where indicated. Data sets containing three or more groups were

401 analyzed by one-way analysis of variance with Bonferroni post-hoc test. Survival curves were compared

402 by Gehan-Breslow-Wilcoxon test. CFU counts were $\log _{10}$-transformed prior to analysis. A $p$ value below

$403 \quad 0.05$ was considered significant. Data represent mean \pm SEM. 
bioRxiv preprint doi: https://doi.org/10.1101/2021.04.21.440820; this version posted April 22, 2021. The copyright holder for this preprint (which

was not certified by peer review) is the author/funder, who has granted bioRxiv a license to display the preprint in perpetuity. It is made available under aCC-BY-NC-ND 4.0 International license.

Data availability:

406 All data generated during the current study are available from the corresponding author on reasonable

407 request.

408 
1. World Health Organization (2018) Tuberculosis: WHO fact sheet no. 104. in Global Tuberculosis Report.

2. S. L. Klein \& K. L. Flanagan, Sex differences in immune responses. Nature Reviews Immunology $16: 626$ (2016).

3. J. A. Seddon, S. S. Chiang, H. Esmail, \& A. K. Coussens, The Wonder Years: What Can Primary School Children Teach Us About Immunity to Mycobacterium tuberculosis? Frontiers in Immunology 9 (2946) (2018).

4. F. Guerra-Silveira \& F. Abad-Franch, Sex bias in infectious disease epidemiology: patterns and processes. PLoS One 8 (4):e62390 (2013).

5. M. Chan-Yeung, K. Noertjojo, S. L. Chan, \& C. M. Tam, Sex differences in tuberculosis in Hong Kong. Int J Tuberc Lung Dis 6 (1):11-18 (2002).

6. J. Y. Feng, et al., Gender differences in treatment outcomes of tuberculosis patients in Taiwan: a prospective observational study. Clinical microbiology and infection : the official publication of the European Society of Clinical Microbiology and Infectious Diseases 18 (9):E331-337 (2012).

7. W. Tan, et al., Sex influences the association between haemostasis and the extent of lung lesions in tuberculosis. Biology of sex differences 9 (1):44 (2018).

8. N. M. Chaves Torres, J. J. Quijano Rodríguez, P. S. Porras Andrade, M. B. Arriaga, \& E. M. Netto, Factors predictive of the success of tuberculosis treatment: A systematic review with metaanalysis. PLoS One 14 (12):e0226507 (2019).

9. J. Dibbern, L. Eggers, \& B. E. Schneider, Sex differences in the C57BL/6 model of Mycobacterium tuberculosis infection. Sci Rep 7 (1):10957 (2017).

10. E. I. Bini, et al., The influence of sex steroid hormones in the immunopathology of experimental pulmonary tuberculosis. PLoS One 9 (4):e93831 (2014).

11. Y. Yamamoto, H. Saito, T. Setogawa, \& H. Tomioka, Sex differences in host resistance to Mycobacterium marinum infection in mice. Infection and immunity 59 (11):4089-4096 (1991).

12. K. Tsuyuguchi, et al., Effect of oestrogen on Mycobacterium avium complex pulmonary infection in mice. Clin Exp Immunol 123 (3):428-434 (2001).

13. J. van Lunzen \& M. Altfeld, Sex differences in infectious diseases-common but neglected. J Infect Dis 209 Suppl 3:S79-80 (2014).

14. M. M. Rosado, E. Bennici, F. Novelli, \& C. Pioli, Beyond DNA repair, the immunological role of PARP-1 and its siblings. Immunology 139 (4):428-437 (2013).

15. B. A. Gibson \& W. L. Kraus, New insights into the molecular and cellular functions of poly(ADPribose) and PARPs. Nat Rev Mol Cell Biol 13 (7):411-424 (2012).

16. L. Liu, et al., Lipopolysaccharide activates ERK-PARP-1-RelA pathway and promotes nuclear factor-kappaB transcription in murine macrophages. Human immunology 73 (5):439-447 (2012).

17. L. Liaudet, et al., Activation of poly(ADP-Ribose) polymerase-1 is a central mechanism of lipopolysaccharide-induced acute lung inflammation. Am J Respir Crit Care Med 165 (3):372-377 (2002).

18. B. Vuong, et al., NF-kappaB transcriptional activation by TNFalpha requires phospholipase C, extracellular signal-regulated kinase 2 and poly(ADP-ribose) polymerase-1. Journal of neuroinflammation 12:229 (2015).

19. K. K. David, S. A. Andrabi, T. M. Dawson, \& V. L. Dawson, Parthanatos, a messenger of death. Frontiers in bioscience (Landmark edition) 14:1116-1128 (2009). 
20. M. F. Langelier, L. Zandarashvili, P. M. Aguiar, B. E. Black, \& J. M. Pascal, NAD(+) analog reveals PARP-1 substrate-blocking mechanism and allosteric communication from catalytic center to DNA-binding domains. Nature communications 9 (1):844 (2018).

21. A. F. Swindall, J. A. Stanley, \& E. S. Yang, PARP-1: Friend or Foe of DNA Damage and Repair in Tumorigenesis? Cancers (Basel) 5 (3):943-958 (2013).

22. E. Matta, A. Kiribayeva, B. Khassenov, B. T. Matkarimov, \& A. A. Ishchenko, Insight into DNA substrate specificity of PARP1-catalysed DNA poly(ADP-ribosyl)ation. Sci Rep 10 (1):3699 (2020).

23. X. Ba \& N. J. Garg, Signaling mechanism of poly(ADP-ribose) polymerase-1 (PARP-1) in inflammatory diseases. Am J Pathol 178 (3):946-955 (2011).

24. Y. Ke, et al., The Role of PARPs in Inflammation-and Metabolic-Related Diseases: Molecular Mechanisms and Beyond. Cells 8 (9) (2019).

25. P. Bai \& L. Virag, Role of poly(ADP-ribose) polymerases in the regulation of inflammatory processes. FEBS Lett 586 (21):3771-3777 (2012).

26. V. Giansanti, F. Dona, M. Tillhon, \& A. I. Scovassi, PARP inhibitors: new tools to protect from inflammation. Biochemical pharmacology 80 (12):1869-1877 (2010).

27. J. Tower, Mitochondrial maintenance failure in aging and role of sexual dimorphism. Archives of biochemistry and biophysics 576:17-31 (2015).

28. N. A. Berger, et al., Opportunities for the repurposing of PARP inhibitors for the therapy of nononcological diseases. British journal of pharmacology 175 (2):192-222 (2018).

29. J. G. Mabley, et al., Gender differences in the endotoxin-induced inflammatory and vascular responses: potential role of poly(ADP-ribose) polymerase activation. J Pharmacol Exp Ther 315 (2):812-820 (2005).

30. D. A. Nusinow, et al., Poly(ADP-ribose) polymerase 1 is inhibited by a histone H2A variant, MacroH2A, and contributes to silencing of the inactive X chromosome. J Biol Chem 282 (17):12851-12859 (2007).

31. B. Stamova, et al., The X-chromosome has a different pattern of gene expression in women compared with men with ischemic stroke. Stroke 43 (2):326-334 (2012).

32. T. Zaremba, et al., Poly(ADP-ribose) polymerase-1 (PARP-1) pharmacogenetics, activity and expression analysis in cancer patients and healthy volunteers. Biochem J 436 (3):671-679 (2011).

33. F. Zhang, et al., Poly(ADP-ribose) polymerase 1 is a key regulator of estrogen receptor alphadependent gene transcription. J Biol Chem 288 (16):11348-11357 (2013).

34. F. Liu, et al., Sex differences in the response to poly(ADP-ribose) polymerase-1 deletion and caspase inhibition after stroke. Stroke 42 (4):1090-1096 (2011).

35. J. A. Calvo, et al., Parp1 protects against Aag-dependent alkylation-induced nephrotoxicity in a sex-dependent manner. Oncotarget 7 (29):44950-44965 (2016).

36. C. Charriaut-Marlangue, et al., Sex differences in the effects of PARP inhibition on microglial phenotypes following neonatal stroke. Brain, behavior, and immunity 73:375-389 (2018).

37. H. Hagberg, et al., PARP-1 gene disruption in mice preferentially protects males from perinatal brain injury. J Neurochem 90 (5):1068-1075 (2004).

38. A. Weaver \& E. Yang, Beyond DNA Repair: Additional Functions of PARP-1 in Cancer. Frontiers in Oncology 3 (290) (2013).

39. S. A. Andrabi, et al., Poly(ADP-ribose) polymerase-dependent energy depletion occurs through inhibition of glycolysis. Proc Natl Acad Sci U S A 111 (28):10209-10214 (2014).

40. Z. Q. Wang, et al., Mice lacking ADPRT and poly(ADP-ribosyl)ation develop normally but are susceptible to skin disease. Genes \& development 9 (5):509-520 (1995).

41. F. Laudisi, M. Sambucci, \& C. Pioli, Poly (ADP-ribose) polymerase-1 (PARP-1) as immune regulator. Endocr Metab Immune Disord Drug Targets 11 (4):326-333 (2011). 
42. A. E. Abdalla, N. Lambert, X. Duan, \& J. Xie, Interleukin-10 Family and Tuberculosis: An Old Story Renewed. International journal of biological sciences 12 (6):710-717 (2016).

43. P. O. Flores-Villanueva, et al., A functional promoter polymorphism in monocyte chemoattractant protein-1 is associated with increased susceptibility to pulmonary tuberculosis. J Exp Med 202 (12):1649-1658 (2005).

44. K. Mohareer, S. Asalla, \& S. Banerjee, Cell death at the cross roads of host-pathogen interaction in Mycobacterium tuberculosis infection. Tuberculosis (Edinb) 113:99-121 (2018).

45. J. D. Ernst, The immunological life cycle of tuberculosis. Nature reviews. Immunology 12 (8):581591 (2012).

46. F. Aredia \& A. I. Scovassi, Poly(ADP-ribose): a signaling molecule in different paradigms of cell death. Biochemical pharmacology 92 (1):157-163 (2014).

47. C. S. Siegel \& L. D. McCullough, NAD+ and nicotinamide: sex differences in cerebral ischemia. Neuroscience 237:223-231 (2013).

48. R. H. Wright, et al., CDK2-dependent activation of PARP-1 is required for hormonal gene regulation in breast cancer cells. Genes \& development 26 (17):1972-1983 (2012).

49. F. J. Oliver, et al., Resistance to endotoxic shock as a consequence of defective NF-kappaB activation in poly (ADP-ribose) polymerase-1 deficient mice. The EMBO journal 18 (16):44464454 (1999).

50. L. Miller (2010) Analyzing gels and western blots with ImageJ.

51. A. A. Ordonez, et al., Radioiodinated DPA-713 imaging correlates with bactericidal activity of tuberculosis treatments in mice. Antimicrob Agents Chemother 59 (1):642-649 (2015). 
527 This work was supported by the JPB foundation and National Institute of Health (NIH) grants Al 137659,

528 Al 37856, Al 130595, HL 133190, HL 140812, P50 NS 38377 and 1RF1 AG 059686. T.M.D. is the Leonard

529 and Madlyn Abramson Professor in Neurodegenerative Diseases. We thank Elizabeth Ihms, DVM, PhD,

530 DACVP (Dane \& Dutchy LLC) and Chris Thoburn (SKCCC Immune Monitoring Core) for helpful discussions

531 and assistance with histopathology and for cytokine/chemokine analysis, respectively.

534 Author Contributions:

535 W.R.B, V.L.D. and S.K. conceived the project. W.R.B., S.K., V.L.D., B.K., S.K.J. and A.A.O. designed the 536 experiments. S.K., A.A.O. and M.K. carried out experiments. T.M.D., V.L.D. and B.K. provided critical 537 reagents and technical expertise. S.K. analyzed the data and prepared the tables and figures. W.R.B and

538 S.K. interpreted the data and wrote the manuscript. All authors read, provided feedback on, and approved 539 the manuscript.

544 The authors declare no competing interests. 
Figure Legends:

(a) Representative PAR immunoblot and (b) densitometric analysis of lung PAR levels in uninfected and implantation: $2.74 \log _{10} \mathrm{CFU}$ ). Each lane represents an individual mouse. Uninfected controls matched the age of mice at $60 \mathrm{dpi}$. Box plots illustrate PAR intensity relative to the uninfected average after normalizing to $ß$-actin $(n=4-5)$. The central line represents the median, the box extends from the $25^{\text {th }}$ to $75^{\text {th }}$ percentiles, and the whiskers represent the range ( $\min$ to max). Mean PAR intensity in infected lungs is 19.92 -fold and 78.34-fold that of uninfected lungs at 60 and $120 \mathrm{dpi}$, respectively. ${ }^{*}, p=0.016$ by unpaired t-test with Welch's correction for unequal variance. ${ }^{* *}, p=0.0059$ by unpaired t test. (c-d)

557 Representative PAR immunoblots showing lung PAR in age-matched uninfected or $M$. $t b$-infected (c) C57BL/6J, (d) 129S2/SvPasCrl or (e) BALB/c mice. Each lane represents an individual mouse. C57BL/6J and 129S2/SvPasCrl mice were infected for 51 days (lung implantation: $1.85 \log _{10}$ CFU) and BALB/c mice for 60 days (lung implantation: $2.74 \log _{10}$ CFU). PAR levels are consistently elevated in infected compared to uninfected lungs.

\section{Fig. 2: PARP inhibitor talazoparib reduces TB-induced PAR formation in mouse lungs.}

(a) Schematic overview of study assessing the effects of short-course PARP inhibition on TB-induced PAR formation. Female C3HeB/FeJ or BALB/c mice (10-12 weeks old) were aerosol-infected with $M$. tb H37Rv (implantation: $2.95 \log _{10}$ CFUs) and treated with the PARP1/2 inhibitor talazoparib or vehicle for 5

567 consecutive days by oral gavage before the lungs were collected for PAR analysis. C3HeB/FeJ mice were 568 treated from 35 to $40 \mathrm{dpi}$ and BALB/c mice from 55 to $60 \mathrm{dpi}$. (b) Representative PAR immunoblots and

570 after 5 days of vehicle (Veh; $264 \mu \mathrm{l} / \mathrm{kg}$ DMSO), low dose (lo; $0.33 \mathrm{mg} / \mathrm{kg}$ ) or high dose (hi; $1 \mathrm{mg} / \mathrm{kg}$ )

571 talazoparib (Tp) administration by oral gavage. Each lane and data point correspond to an individual

572 mouse. PAR levels were normalized to ß-actin and are expressed relative to the average intensity in 573 vehicle-treated mice. ${ }^{*}, p=0.0231$ by unpaired t-test. The PARP1/2 inhibitor talazoparib reduced lung

574 PAR levels in M. tb-infected mice in a dose-dependent manner. 
Figure 3. PARP1 deletion abolishes sex differences in TB immune responses.

577

578

579

580

581

582

583

584

Induction of key cytokines and chemokines in the lungs of male and female (a) WT (blue) and (b) PARP1 /- (red) mice 28 days after aerosol infection with M. tb H37Rv (implantation: $1.86 \log _{10}$ CFU). Lung cyto/chemokines were quantified by Luminex multiplex assay and are expressed as fold change relative to levels in uninfected lungs $(n=2-3)$. Values above 1 indicate levels that are higher in infected than in uninfected lungs. ${ }^{*}, p<0.05$ by unpaired t-test. PARP1 deletion reduced the induction of TNF $\alpha$, IFNץ, IL6, IL-1 13 and MCP-1 in male mice to female levels and eliminated the sex differences observed in WT mice.

\section{Figure 4. PARP1 deletion dampens proinflammatory responses in male but not in female mice.}

Change in lung cytokines and chemokines over time in $(a, c)$ male or $(b, d)$ female WT (blue) or PARP1 ${ }^{-/}$ (red) mice following aerosol infection with M. tb H37Rv (implantation: $1.86 \log _{10}$ CFU). Lung cytokines were quantified by Luminex multiplex assay and are expressed as fold change relative to levels in uninfected lungs ( $n=2-3$ ). Values above 1 indicate levels that are higher in infected than in uninfected lungs. PARP1 deletion dampened IL-1ß, TNF $\alpha$, IFN $Y$ and MCP-1 responses following $M$. th infection in males but not in females but eliminated IL-10 induction in both male and female mice.

\section{Figure 5. PARP1 deletion prolongs TB survival in female mice.}

TB survival in WT (blue) or PARP1 ${ }^{--}$(red) mice aerosol-infected with M. tb H37Rv. (a) Day 1 lung implantation (mean: $2.397 \log _{10}$ CFUs). (b-c) Survival analysis in $M . t b$-infected female (b; $\left.n=10-15\right)$ or male ( $c$; $n=15$ ) WT or PARP1 ${ }^{-/}$mice Statistical differences in survival were assessed by Gehan-BreslowWilcoxon test. PARP1 $\%$ females survive infection significantly longer than WT females.

\section{Figure 6. Bacterial containment is improved in female but impaired in male PARP1-deficient mice.}

Bacterial burden in WT (blue) or PARP1\% (red) mice aerosol-infected with M. tb H37Rv. (a) Lung (left) and spleen (right) bacterial burden in female or male WT or PARP1 ${ }^{--}$mice (day $1: 1.86 \log _{10} \mathrm{CFU}$ ). Each point represents the mean bacterial burden of 2-3 male or female mice. ${ }^{*}, p<0.05$ by unpaired t-test (lung CFUs: male vs. female PARP1 ${ }^{--}$mice). (b-c) Lung bacterial burden in WT (blue; $+/+$) and PARP1 ${ }^{--}$ 
604

605

606

607

608

609

610

611

612

613

614

615

616

617

618

619

620

621

622

623

624

625

626

627

628

629

630

(red; -/-) mice after (b) 1 or (c) 3 months of infection with M. tb H37Rv or H37Rv $\Delta p n c A$. Data was collected in three independent experiments (day 1: 1.659 (0.3172), 1.724 (0.0916) and 1.858 (0.2356) $\log _{10}$ CFU (SD)). Horizontal lines represent the mean +/- SEM. ${ }^{*}, p<0.05 ; * *, p<0.01$ by unpaired t-test. PARP1 deficiency improves bacterial containment in females but impairs it in male mice.

\section{Figure 7. PARP1 deletion exacerbates TB lung inflammation in male mice.}

(a) Formalin-fixed lungs of male (M) and female (F) WT or PARP1 ${ }^{-1}$ mice 3 months after infection with $M$. tb H37Rv (lung implantation: $1.66 \log _{10}$ CFU). (b) Fresh (unfixed) lung weights of male and female WT (blue) or PARP1 $\%$ (red) mice 3 months after $M$. tb infection (circle) or of age-matched uninfected controls (cross). (c) Corresponding body weights of the infected mice depicted in (b). ${ }^{*}, p<0.05 ;{ }^{* * *}, p$ $<0.001$ by student's t-test. The lungs of infected, but not uninfected, PARP1 ${ }^{--}$males are significantly larger than those of any other group, despite them having a lower total body weight than WT males. (d) Representative H\&E-stained lung sections of male and female WT or PARP1 ${ }^{\%-}$ mice 3 months after infection with M. tb H37Rv (lung implantation: $1.66 \log _{10} \mathrm{CFU}$ ). There was extensive consolidation throughout the lungs, in particular in PARP1 ${ }^{-/}$males. (e-f) Quantification of area of consolidation (e) and percent lung involvement (f) indicating lung inflammation. Each point represents an individual mouse. One-way analysis of variance with Bonferroni post-test; ${ }^{*}, p<0.05 ;{ }^{* *}, p<0.01$. PARP1 ${ }^{-/}$males have significantly more lung inflammation than females or WT males in chronic TB.

\section{Figure 8. Proposed model: PARP1 drives TB sex differences.}

PARP1 is activated by $M . t b$ infection and interacts with numerous transcription factors, such as ERK2, NF-KB and AP-1, to shape the TB host response. PARP1 drives exaggerated inflammatory responses in males that enhance their ability to contain the infection. In contrast, PARP1 antagonizes TB containment in females by promoting primarily immunosuppressive IL-10 responses. As a result, PARP1 deletion increases TB resistance in female mice but exacerbates inflammation in male mice. 


\section{Supplemental Figures:}

632

633

634

635

636

637

638

639

640

641

642

643

644

645

646

647

648

649

650

651

652

653

654

655

656

657

658

\section{Figure S1. Confirmation of PARP1 deletion in 129S-Parp1 $1^{\text {tm1Zqw }} / \mathrm{J}$ mice.}

(a) PARP1 genotyping PCR products of two representative 129S1/SvImJ (WT) and two 129S-Parp1 $1^{\text {tm1Zqw}} / \mathrm{J}$ $\left(\right.$ PARP1 $^{-1}$ ) mice separated on a $1.2 \%$ agarose gel. Expected size is $112 \mathrm{bp}$ for the wild type and $350 \mathrm{bp}$ for the PARP1 mutant band. L, Low mass DNA ladder. NTC, no target control. (b) PARP1 Western blot of five 129S1/SvImJ (left) and five 129S-Parp1 $1^{\text {tm1Zqw }} / \mathrm{J}$ (right) mice demonstrating no detectable PARP1 protein (116 kDa) in the knockout mice.

Figure S2. Lung levels of cytokines and chemokines in uninfected WT and PARP1 ${ }^{\%}$ mice do not differ by sex.

Levels of lung cyto-/chemokine in uninfected male and female (a) WT or (b) PARP1\% (red) mice, quantified by Luminex multiplex assay $(n=2)$. At baseline, cytokine and chemokine levels are unaffected by sex of PARP1 deletion.

\section{Figure S3. Not all TB immune responses are affected by PARP1 ${ }^{-/}$status.}

Change in lung cyto-/chemokine levels in WT (blue) and PARP1 ${ }^{\%-}$ (red) mice (a-b) 28 or (c-d) 74 days after aerosol infection with $M . t b \mathrm{H} 37 \mathrm{Rv}(\mathrm{n}=2-3)$. Cyto-/Chemokine levels were quantified by Luminex multiplex assay and are expressed as fold change relative to levels in uninfected lungs (set to 1 ; time 0 , dashed line). Values above 1 indicate levels that are higher in infected than uninfected lungs whereas values less than 1 indicate levels that are reduced in infected lungs. The levels of IL-2, IL-12, IL-17 and MIP-1 $\alpha$ were largely unaffected by $M$. tb infection, PARP1 status or sex, and at $74 \mathrm{dpi}$, most TB immune responses were unaffected by PARP1 deletion or sex .

\section{Figure S4. Chronically TB-infected male but not female PARP1 $\%$ mice have enlarged lungs.}

(a) Lung pictures of male (M) and female (F) wildtype 129S1/SvImJ (WT) or 129S-Parp1 ${ }^{\text {tm1Zqw }} /$ J $^{\text {PARP1 }}{ }^{-/}$) mice at 21, 28, 42, 56 and 74 days post aerosol infection with M. tb H37Rv. At days 28, 56 and 74, only the left lobes (pictured) were collected for gross pathology and CFU determination and the right lobes immediately processed for cytokine and PAR level analysis. (b-d) Average weight of lungs (b-c) or mice 
(d) 74 days post infection or of age-matched uninfected mice (c). ${ }^{*}, p<0.05$; n.s., not significant by oneway ANOVA with Bonferroni post test.

Figure S5. Histopathology reveals extensive lung consolidation in PARP1 ${ }^{-/}$males.

H\&E-stained lung sections of all male and female WT or PARP1\% mice 3 months after infection with $M$. tb H37Rv (day 1: $1.66 \log _{10} \mathrm{CFU}$ ). There was extensive consolidation throughout the lungs, in particular in PARP1 ${ }^{--}$males. Quantification of consolidation and percent lung involvement indicating lung inflammation shown in figure 4.

\section{Figure S6. Inflammation analysis by ${ }^{18} \mathrm{~F}$-FDG PET-CT.}

Inflammation analysis in 3 male and 3 female WT or PARP1 ${ }^{-/}$mice 3 months after infection with M. $t b$ H37Rv (day 1: $1.66 \log _{10}$ CFU) by CT (a) and ${ }^{18}$ F-FDG PET (b). Each data point represents the mean activity recorded in the left or the right lung ( 2 data points per mouse). (a) CT values of $-1000,0$ and $+1000 \mathrm{HU}$

672 indicate air, water and bone, respectively. CT values above $0 \mathrm{HU}$ suggest diseased lung. (b) Mean ${ }^{18} \mathrm{~F}-$

673 FDG PET activity per lung was normalized to the injected dose and body weight. SUV = Standardized

674 uptake value. (c) For each mouse, lung bacterial burden was plotted against CT (average of left and right 675 lung). CT values correlate with bacterial burden.

678 (a-b) Time course of bacterial burden in the (a) lungs and (b) spleens of male and female 129S1/SvImJ 679 WT mice aerosol-infected with $M$. tb H37Rv or H37RvApncA. Data was collected in three independent experiments (day 1: $1.659 \pm 0.3172,1.724 \pm 0.0916$ and $1.858 \pm 0.2356 \log _{10}$ CFU $( \pm$ SD)). Each data point represents an individual mouse. Lines indicate mean and SEM. (c) Survival in male and female 129S1/SvImJ WT mice ( $n=15$ ) following aerosol infection with M. tb H37Rv (day 1: $2.397 \log _{10}$ CFU). (d)

683 Lung inflammation in male and female 129S1/SvIm WT mice 3 months after aerosol infection with $M$. tb 684 H37Rv (day 1: $1.66 \log _{10}$ CFU). Each point represents the area of consolidation, determined histologically, in an individual mouse. There were no apparent sex differences in bacterial burden, survival or lung inflammation in TB-infected WT mice. 
688 Figure S8. MCP-1 correlates with bacterial burden in WT and PARP1 ${ }^{-1}$ mice.

689 (a-b) MCP-1 levels (a) and bacterial burden (b) in the lungs of male and female WT (blue) and PARP1\%

690 (maroon) mice 28 ad 74 days after aerosol infection with M. tb H37Rv (implantation: $1.86 \log _{10}$ CFU).

691 Lung cytokines were quantified by Luminex multiplex assay. At $28 \mathrm{dpi}$, PARP1\%- females have the lowest

692 MCP-1 levels and lowest bacterial burden, and at $74 \mathrm{dpi}, \mathrm{PARP} 1 \%$ - males have the highest MCP-1 levels

693 and highest bacterial burden. (c) Spearman correlation analysis of bacterial burden vs. MCP-1 levels in

694 WT (blue) and PARP1 ${ }^{--}$(red) mice. Each point represents an individual mouse. $r$, correlation coefficient.

$695 \mathrm{Cl}, 95 \%$ confidence interval. MCP-1 levels correlate with bacterial burden in WT and PARP1 ${ }^{-/}$mice.

696

697

698 
bioRxiv preprint doi: https://doi.org/10.1101/2021.04.21.440820; this version posted April 22, 2021. The copyright holder for this preprint (which

was not certified by peer review) is the author/funder, who has granted bioRxiv a license to display the preprint in perpetuity. It is made available under aCC-BY-NC-ND 4.0 International license.

699

Tables

700

701

\begin{tabular}{|r|c|c|c|c|}
\hline Table 1: Survival statistics following moderate TB infection $\left(2.397 \log _{10} \mathrm{CFU}\right)$. \\
\cline { 2 - 3 } & Median Survival (days) & $\begin{array}{c}\text { Hazard Ratio } \\
(95 \% \mathrm{Cl})\end{array}$ & $\begin{array}{c}\text { Significant? } \\
(p)\end{array}$ \\
\cline { 2 - 3 } Females & $\mathbf{\text { WT }}$ & PARP1 ${ }^{--}$ & $\begin{array}{c}5.225 \\
(1.758-15.52)\end{array}$ & $\begin{array}{c}\text { Yes } \\
(\mathbf{0 . 0 0 3 1})\end{array}$ \\
\hline Males & $\mathbf{9 3}$ & $\mathbf{1 3 4}$ & $\begin{array}{c}0.7030 \\
(0.3248-1.522)\end{array}$ & $\begin{array}{c}\text { No } \\
(0.4942)\end{array}$ \\
\hline Combined & 93 & 109.5 & $\begin{array}{c}\text { No } \\
(0.325\end{array}$ & $(0.1375)$ \\
\hline
\end{tabular}

702 
Fig. 1

a

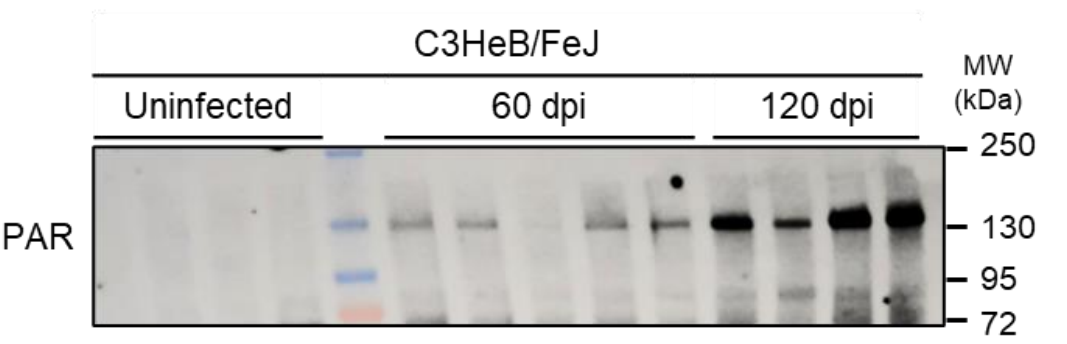

ß-actin

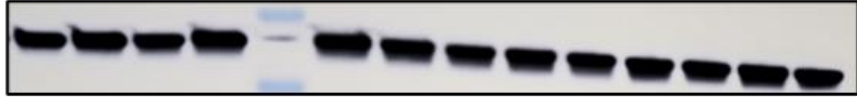

C

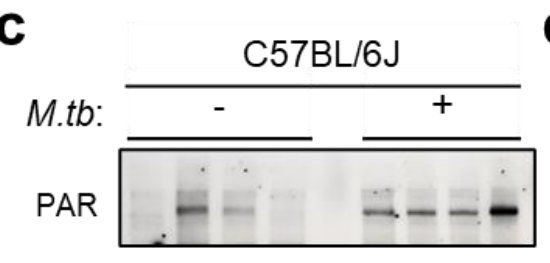

B-Actin
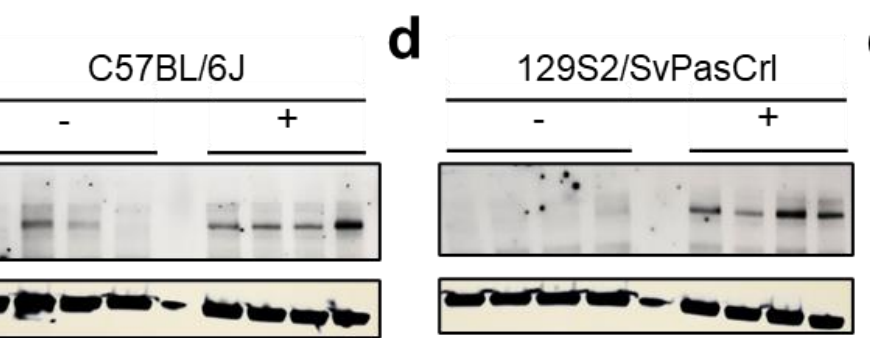

b

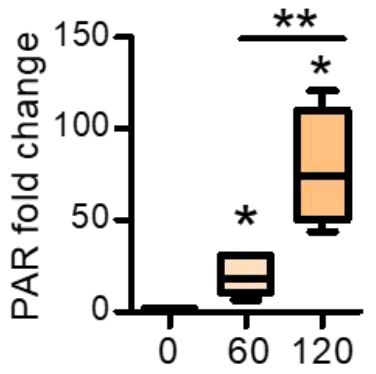

Days post infection

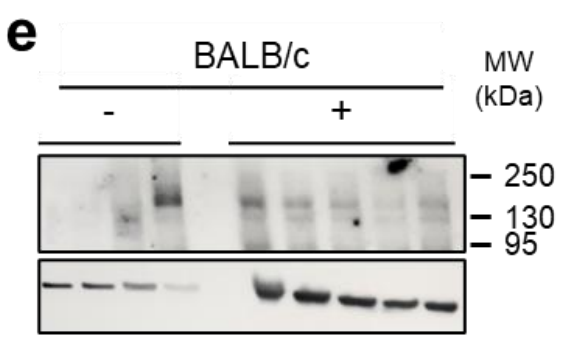

Figure 1. TB infection triggers PARP activation in mouse lungs.

(a) Representative PAR immunoblot and (b) densitometric analysis of lung PAR levels in uninfected and $\mathrm{M}$. tb-infected $\mathrm{C} 3 \mathrm{HeB} / \mathrm{FeJ}$ mice 60 and 120 days post infection (dpi) with aerosolized $\mathrm{M}$. tb H37Rv (lung implantation: $2.74 \log 10 \mathrm{CFU}$ ). Each lane represents an individual mouse. Uninfected controls matched the age of mice at $60 \mathrm{dpi}$. Box plots illustrate PAR intensity relative to the uninfected average after normalizing to $B$-actin $(n=4-5)$. The central line represents the median, the box extends from the 25 th to 75 th percentiles, and the whiskers represent the range ( $\min$ to max). Mean PAR intensity in infected lungs is 19.92 -fold and 78.34 -fold that of uninfected lungs at 60 and $120 \mathrm{dpi}$, respectively. ${ }^{*}, p$ $=0.016$ by unpaired $t$-test with Welch's correction for unequal variance. ${ }^{* \star}, p=0.0059$ by unpaired $t$ test. (c-d) Representative PAR immunoblots showing lung PAR in age-matched uninfected or M. tbinfected (c) C57BL/6J, (d) 129S2/SvPasCrl or (e) BALB/c mice. Each lane represents an individual mouse. C57BL/6J and 129S2/SvPasCrl mice were infected for 51 days (lung implantation: $1.85 \log 10$ $\mathrm{CFU}$ ) and BALB/c mice for 60 days (lung implantation: $2.74 \log 10 \mathrm{CFU}$ ). PAR levels are consistently elevated in infected compared to uninfected lungs. 
a

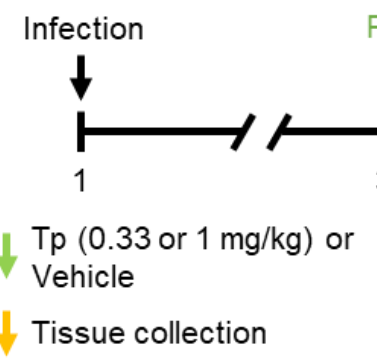

PARP inhibition

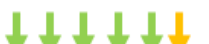

35

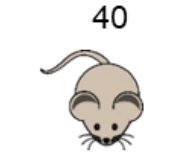

C3HeB/FeJ
PARP inhibition

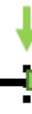

55
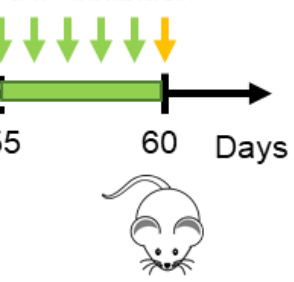

BALB/c

C $\mathrm{C} 3 \mathrm{HeB} / \mathrm{FeJ}$

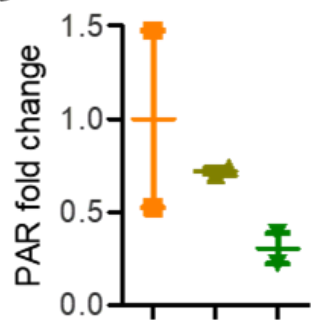

$B A L B / C$

b C3HeB/FeJ

PAR

\begin{tabular}{rr|r|}
\hline Veh & Tp-lo & Tp-hi \\
\hline & \\
\hline
\end{tabular}

ß-Actin

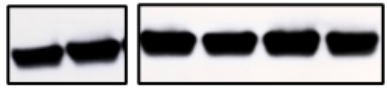

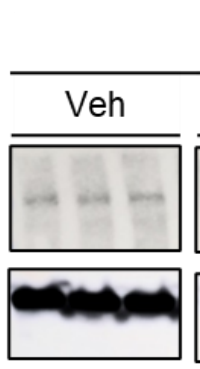

BALB/C

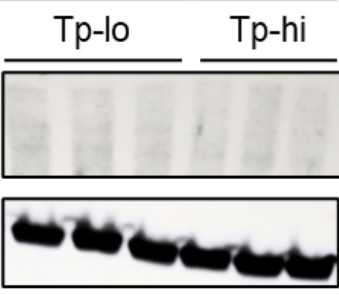

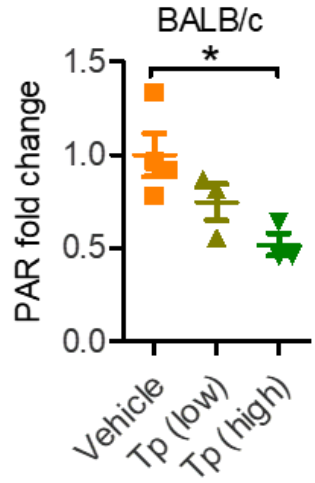

Fig. 2: PARP inhibitor talazoparib reduces TB-induced PAR formation in mouse lungs.

(a) Schematic overview of study assessing the effects of short-course PARP1/2 inhibition on TB-induced PAR formation. Female C3HeB/FeJ or BALB/c mice (10-12 weeks old) were aerosol-infected with M.tb H37Rv (implantation: $2.95 \log _{10} \mathrm{CFUs}$ ) and treated with the PARP1/2 inhibitor talazoparib or vehicle for 5 consecutive days by oral gavage before the lungs were collected for PAR analysis. C3HeB/FeJ mice were treated from 35 to $40 \mathrm{dpi}$ and BALB/c mice from 55 to $60 \mathrm{dpi}$. (b) Representative PAR immunoblots and (c) densitometric analyses of lung PAR levels in TB-infected $\mathrm{C} 3 \mathrm{HeB} / \mathrm{FeJ}$ (40 dpi) and BALB/c mice (60 dpi) after 5 days of vehicle (Veh; $264 \mu \mathrm{l} / \mathrm{kg}$ DMSO), low dose (lo; $0.33 \mathrm{mg} / \mathrm{kg}$ ) or high dose (hi; $1 \mathrm{mg} / \mathrm{kg}$ ) talazoparib (Tp) administration by oral gavage. Each lane and data point correspond to an individual mouse. PAR levels were normalized to $ß$-actin and are expressed relative to the average intensity in vehicle-treated mice. ${ }^{*}, p=0.0231$ by unpaired t-test. The PARP1/2 inhibitor talazoparib reduced lung PAR levels in M.tbinfected mice in a dose-dependent manner. 
Fig. 3

a

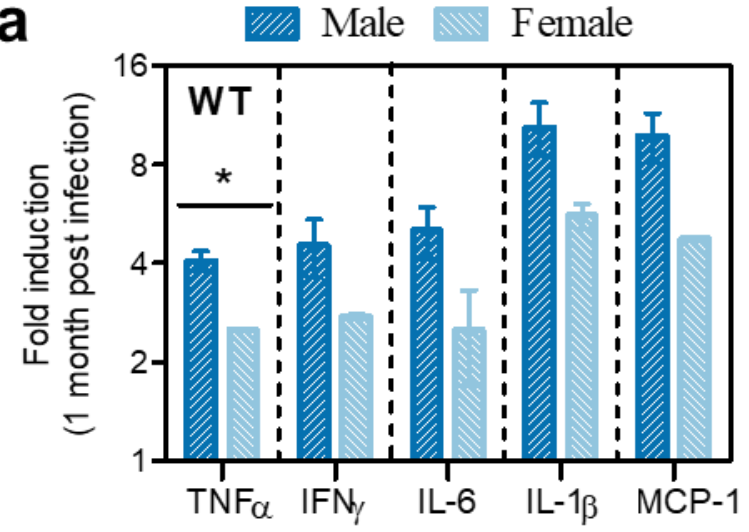

b

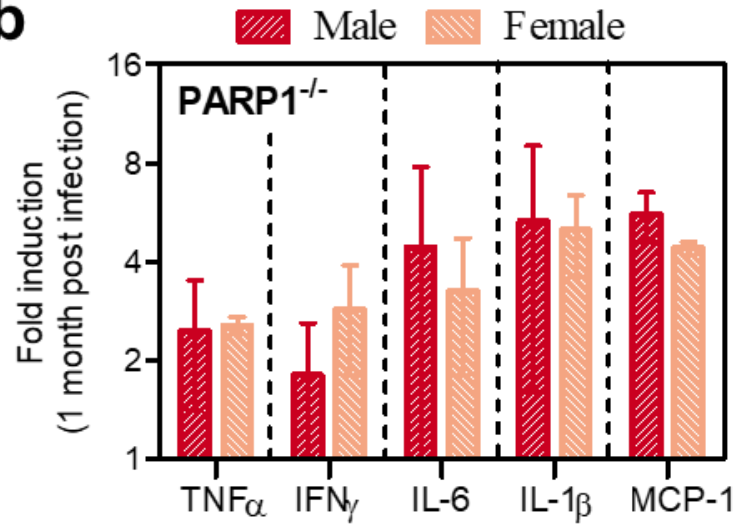

Figure 3. PARP1 deletion abolishes sex differences in TB immune responses.

Induction of key cytokines and chemokines in the lungs of male and female (a) WT (blue) and (b) PARP $1^{-/}$(red) mice 28 days after aerosol infection with M.tb H37Rv (implantation: $1.86 \log _{10}$ CFU). Lung cyto-/chemokines were quantified by Luminex multiplex assay and are expressed as fold change relative to levels in uninfected lungs $(n=2-3)$. Values above 1 indicate levels that are higher in infected than in uninfected lungs. ${ }^{*}, p<0.05$ by unpaired t-test. PARP1 deletion reduced the induction of TNF $\alpha$, IFN $\gamma, I L-6, I L-1 \beta$ and MCP-1 in male mice to female levels and eliminated the sex differences observed in WT mice. 
Fig. 4

a
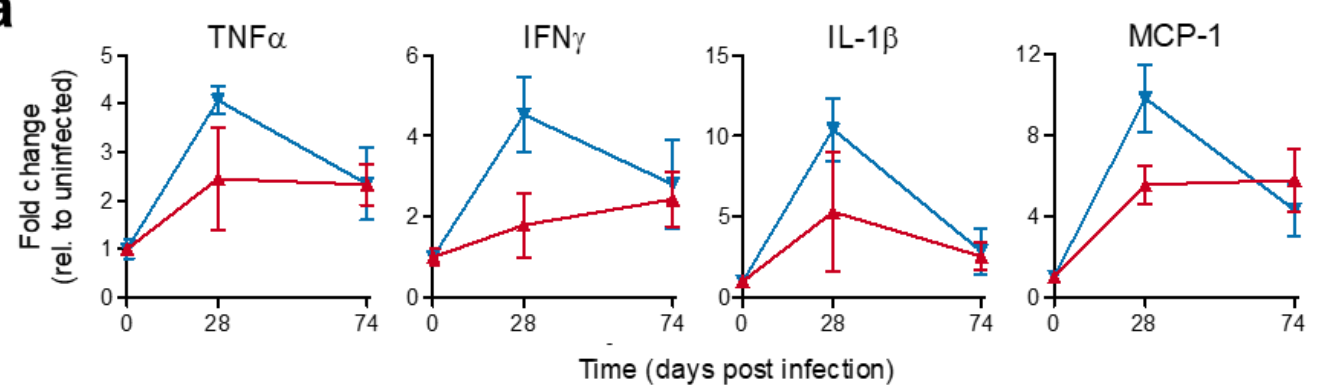

Males

$\rightarrow$ WT

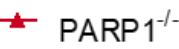

b
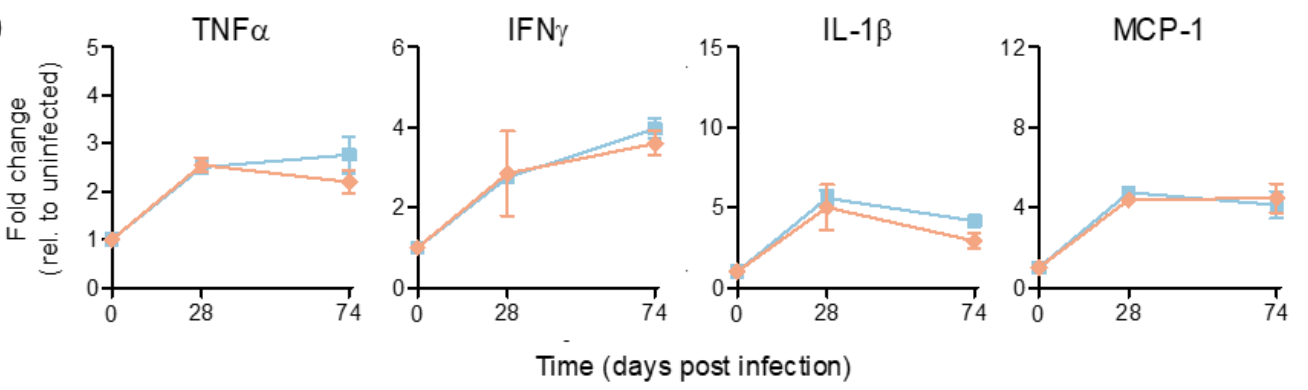

Females

Time (days post infection)

C

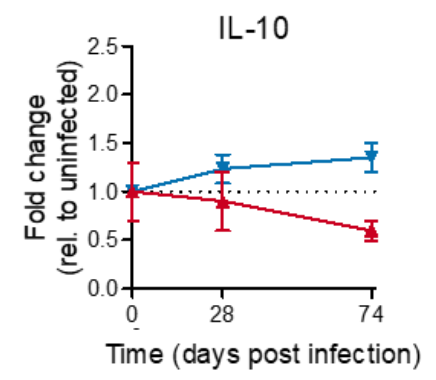

d

\section{Males}

$\rightarrow$ WT

\pm PARP1 $^{-1-}$

Figure 4. PARP1 deletion dampens proinflammatory responses in male but not in female mice.

Change in lung cytokines and chemokines over time in $(\mathbf{a}, \mathbf{c})$ male or $(\mathbf{b}, \mathbf{d})$ female WT (blue) or PARP $1^{-/}$(red) mice following aerosol infection with M.tb H37Rv (implantation: $1.86 \log _{10} \mathrm{CFU}$ ). Lung cytokines were quantified by Luminex multiplex assay and are expressed as fold change relative to levels in uninfected lungs $(n=2-3)$. Values above 1 indicate levels that are higher in infected than in uninfected lungs. PARP1 deletion dampened IL-1ß, TNFa, IFNy and MCP-1 responses following M.tb infection in males but not in females but eliminated IL-10 induction in both male and female mice. 
Fig. 5
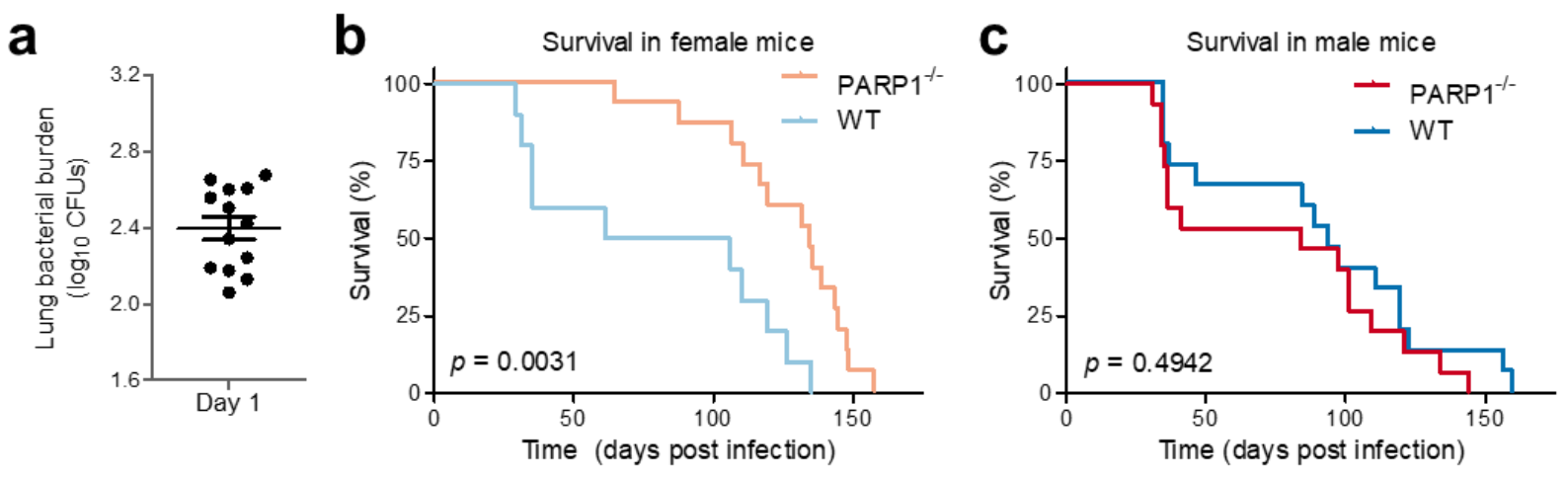

Figure 5. PARP1 deletion prolongs TB survival in female mice.

TB survival in WT (blue) or PARP1-- (red) mice aerosol-infected with M.tb H37Rv. (a) Day 1 lung implantation (mean: $2.397 \log _{10}$ CFUs). (b-c) Survival analysis in M.tb-infected female (b; $\left.n=10-15\right)$ or male $(\mathbf{c} ; n=15)$ WT or PARP1-- mice Statistical differences in survival were assessed by Gehan-Breslow-Wilcoxon test. PARP 1 - females survive infection significantly longer than WT females. 
Fig. 6
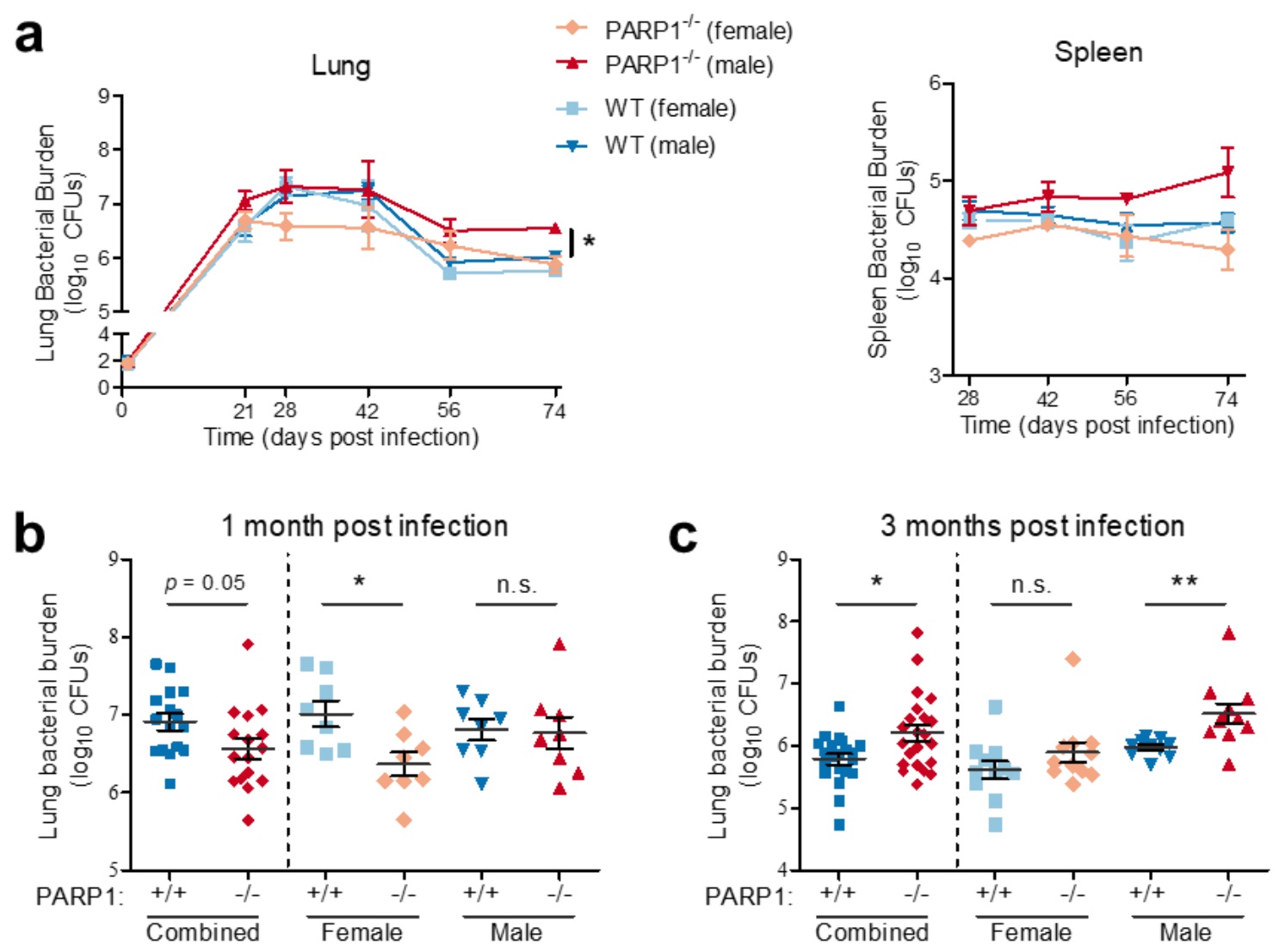

Figure 6. Bacterial containment is improved in female but impaired in male PARP1deficient mice.

Bacterial burden in WT (blue) or PARP1-/ (red) mice aerosol-infected with M.tb H37Rv. (a) Lung (left) and spleen (right) bacterial burden in female or male WT or PARP1/- mice (day $1: 1.86 \log _{10}$ CFU). Each point represents the mean bacterial burden of $2-3$ male or female mice. ${ }^{*}, p<0.05$ by unpaired t-test (lung CFUs: male vs. female PARP1-- mice). (b-c) Lung bacterial burden in WT (blue; +/+) and PARP1-/ (red; -/-) mice after (b) 1 or (c) 3 months of infection with M.tb H37Rv or H37Rv $\Delta$ pncA. Data was collected in three independent experiments (day 1: $1.659(0.3172), 1.724$ $(0.0916)$ and $1.858(0.2356) \log _{10}$ CFU (SD)). Horizontal lines represent the mean $+/-$ SEM. ${ }^{*}, p<$ $0.05 ;{ }^{* *}, p<0.01$ by unpaired t-test. PARP1 deficiency improves bacterial containment in females but impairs it in male mice. 
Fig. 7

a

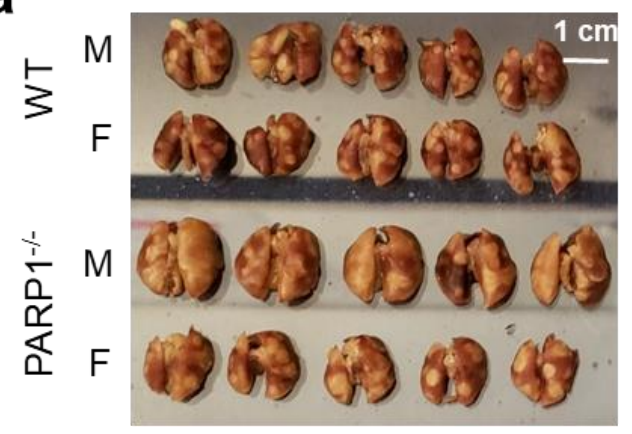

d
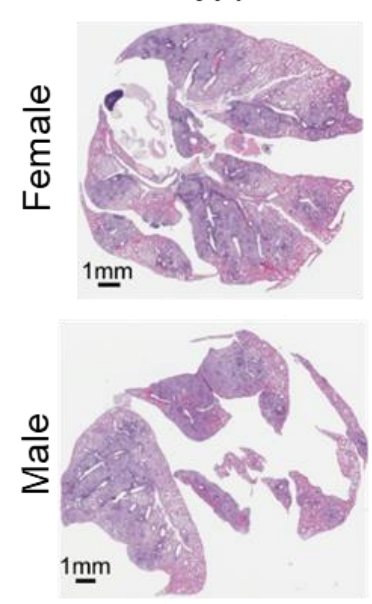

b

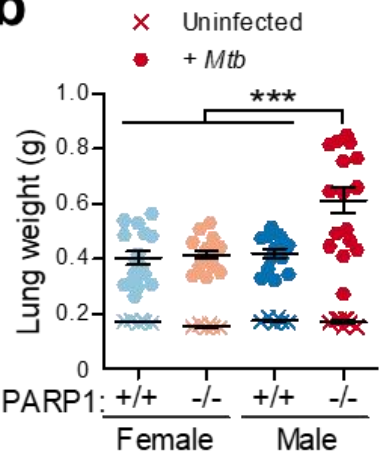

e

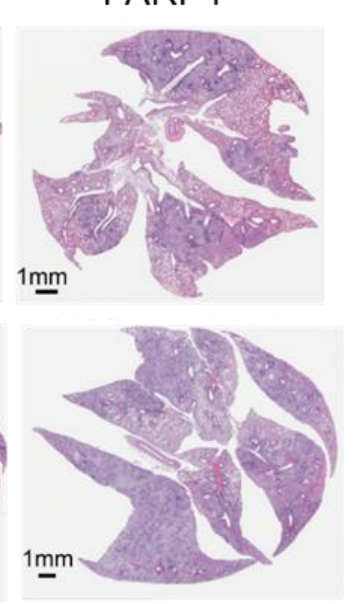

C

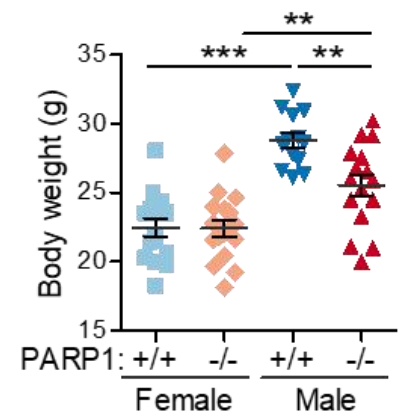

f

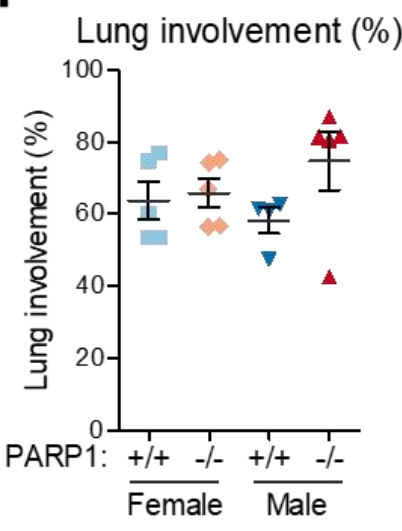

Figure 7. PARP1 deletion exacerbates TB lung inflammation in male mice.

(a) Formalin-fixed lungs of male (M) and female (F) WT or PARP1-1- mice 3 months after infection with M.tb H37Rv (lung implantation: $1.66 \log _{10}$ CFU). (b) Fresh (unfixed) lung weights of male and female WT (blue) or PARP1-1- (red) mice 3 months after M.tb infection (circle) or of age-matched uninfected controls (cross). (c) Corresponding body weights of the infected mice depicted in (b). ${ }^{*}, p<0.05 ;{ }^{* \star *}, p<0.001$ by student's t-test. The lungs of infected, but not uninfected, PARP $1^{-1-}$ males are significantly larger than those of any other group, despite them having a lower total body weight than WT males. (d) Representative H\&E-stained lung sections of male and female WT or PARP 1 - mice 3 months after infection with M.tb H37Rv (lung implantation: $1.66 \log _{10}$ CFU). There was extensive consolidation throughout the lungs, in particular in PARP1-- males. (e-f) Quantification of area of consolidation (e) and percent lung involvement (f) indicating lung inflammation. Each point represents an individual mouse. One-way analysis of variance with Bonferroni post-test; ${ }^{*}, p<0.05 ;{ }^{* *}, p$ $<0.01$. PARP $1^{-1-}$ males have significantly more lung inflammation than females or WT males in chronic TB. 


\section{Fig. 8}
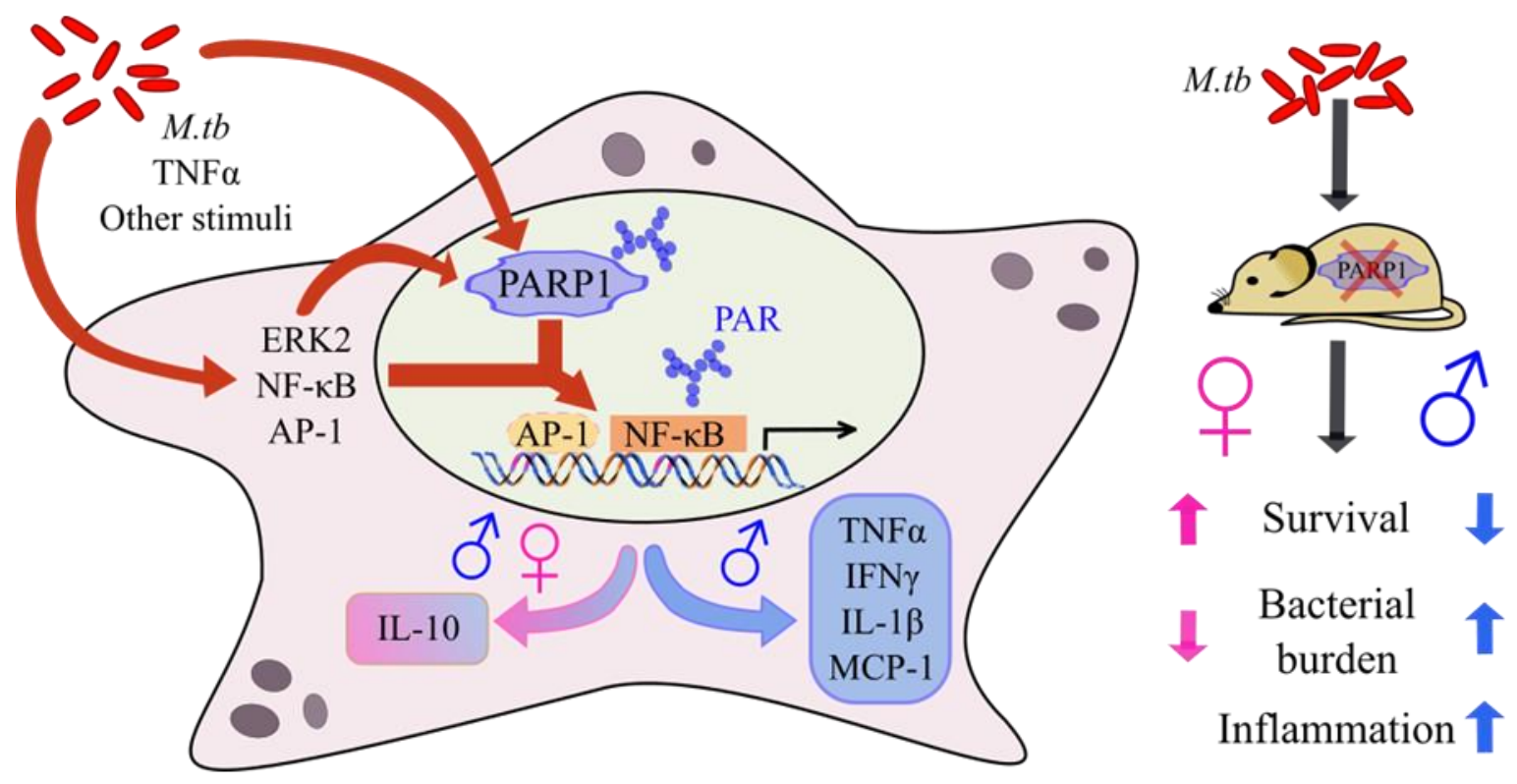

Figure 8. Proposed model: PARP1 drives TB sex differences.

PARP1 is activated by M.tb infection and interacts with numerous transcription factors, such as ERK2, NF-KB and AP-1, to shape the TB host response. PARP1 drives exaggerated inflammatory responses in males that enhance their ability to contain the infection. In contrast, PARP1 antagonizes TB containment in females by promoting primarily immunosuppressive IL-10 responses. As a result, PARP1 deletion increases TB resistance in female mice but exacerbates inflammation in male mice. 


\section{Fig. S1}

a

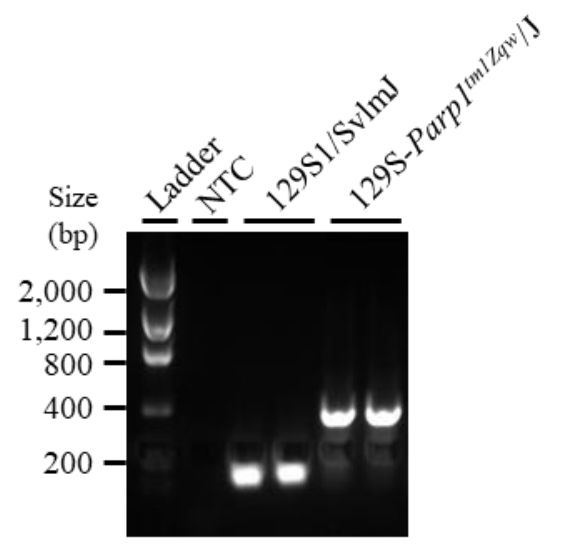

b

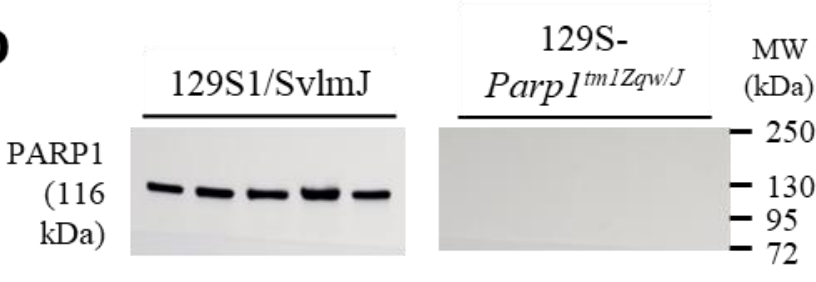

ß-Actin

$(42$

$\mathrm{kDa})$

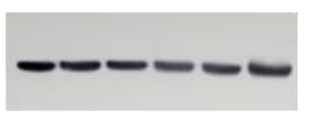

Figure S1. Confirmation of PARP1 deletion in 129SParp1 $^{\text {tm1Zqw } / J ~ m i c e . ~}$

(a) PARP1 genotyping PCR products of two representative 129S1/SvImJ (WT) and two 129S-Parp $1^{\text {tm12qw }} /$ J (PARP1 ${ }^{-/}$) mice separated on a $1.2 \%$ agarose gel. Expected size is $112 \mathrm{bp}$ for the wild type and $350 \mathrm{bp}$ for the PARP1 mutant band. L, Low mass DNA ladder. NTC, no target control. (b) PARP1 Western blot of five 129S1/SvImJ (left) and five 129S-Parp1 ${ }^{\text {tm1Zqw }} / \mathrm{J}$ (right) mice demonstrating no detectable PARP1 protein (116 $\mathrm{kDa}$ ) in the knockout mice. 


\section{Fig. S2}

a

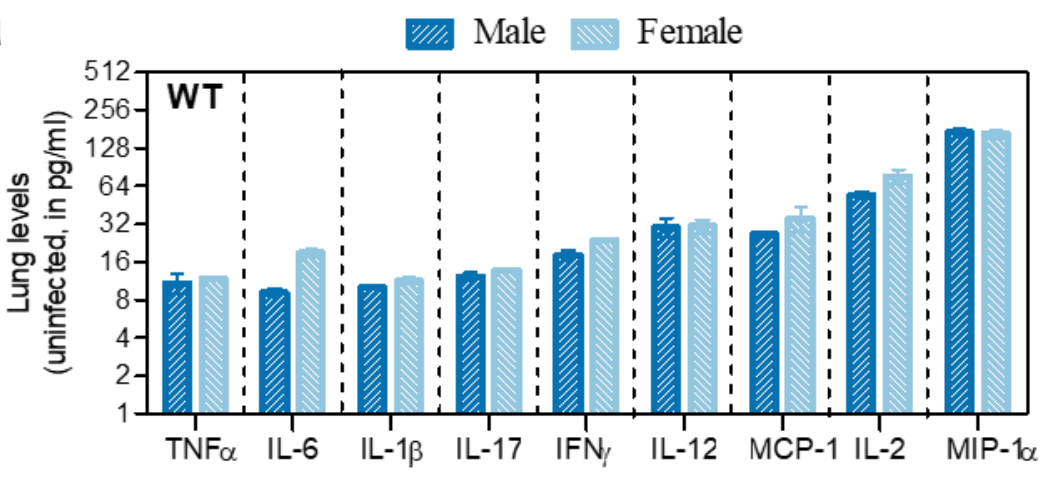

b

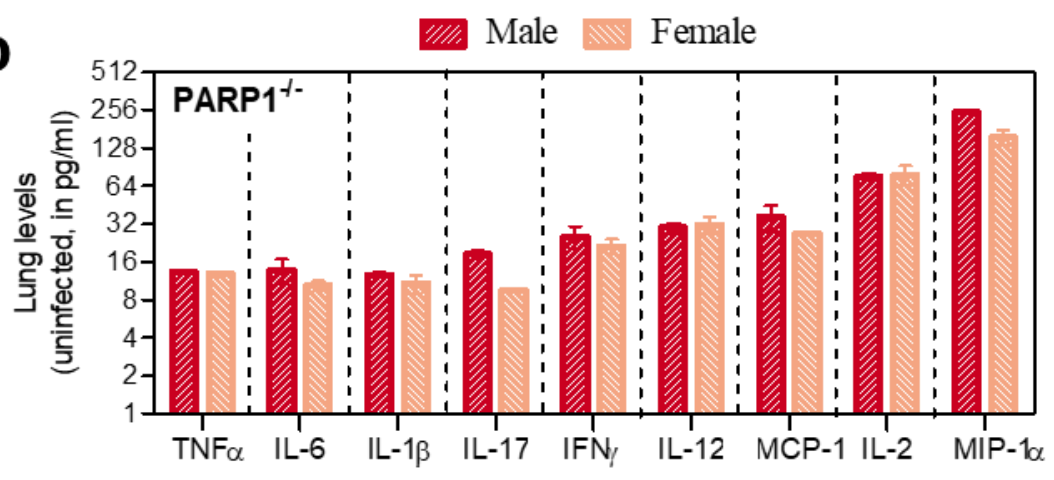

Figure S2. Lung levels of cytokines and chemokines in uninfected WT and PARP1-/- mice do not differ by sex.

Levels of lung cyto-/chemokine in uninfected male and female (a)

WT or (b) PARP1-/ (red) mice, quantified by Luminex multiplex assay $(n=2)$. At baseline, cytokine and chemokine levels are unaffected by sex of PARP1 deletion. 
Fig. S3
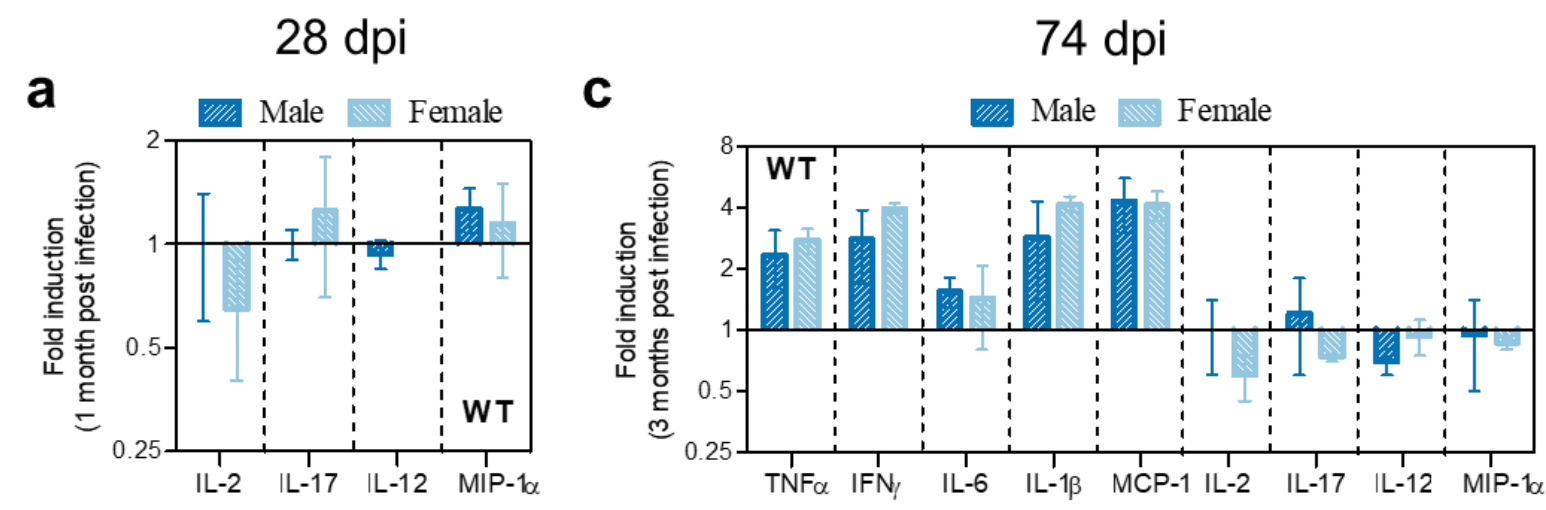

b

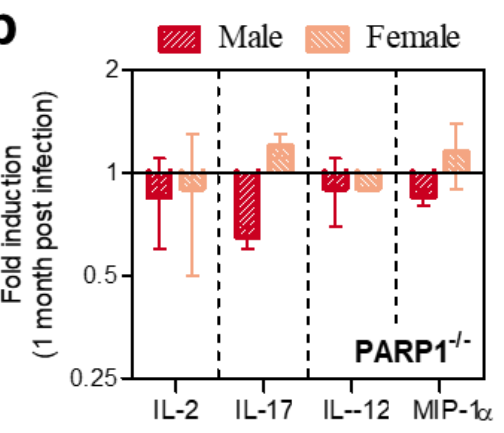

d

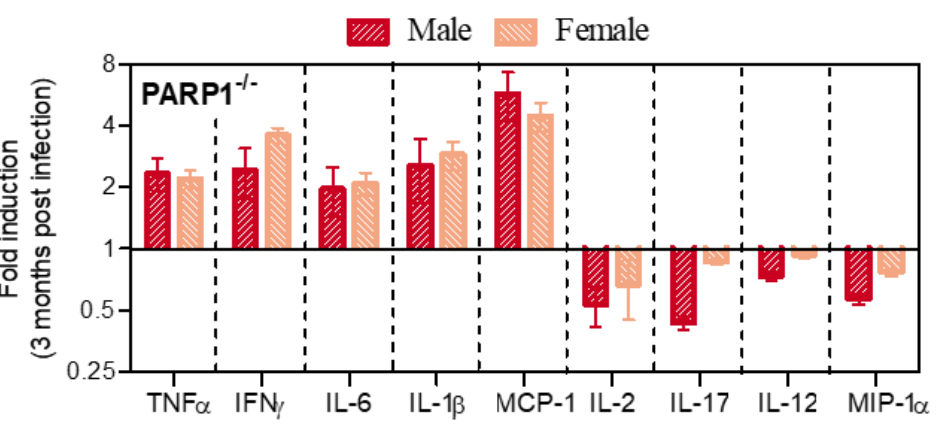

Figure S3. Not all TB immune responses are affected by PARP1-/status.

Change in lung cyto-/chemokine levels in WT (blue) and PARP1-/ (red) mice (a-b) 28 or (c-d) 74 days after aerosol infection with M.tb H37Rv ( $n=2-3)$. Cyto-/Chemokine levels were quantified by Luminex multiplex assay and are expressed as fold change relative to levels in uninfected lungs (set to 1 ; time 0 , dashed line). Values above 1 indicate levels that are higher in infected than uninfected lungs whereas values less than 1 indicate levels that are reduced in infected lungs. The levels of IL-2, IL-12, IL-17 and MIP-1 1 were largely unaffected by M.tb infection, PARP1 status or sex, and at $74 \mathrm{dpi}$, most TB immune responses were unaffected by PARP1 deletion or sex . 
a

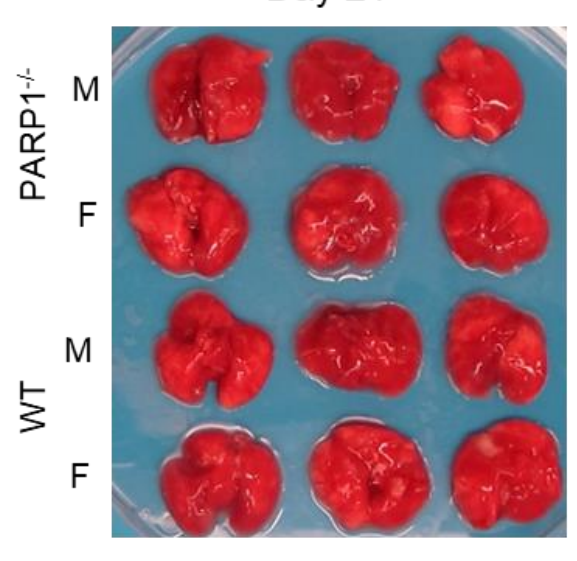

Day 56
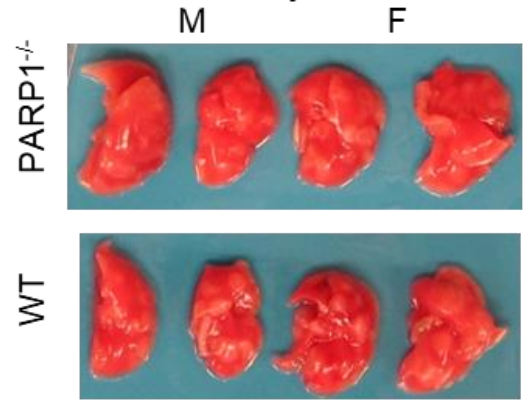

b

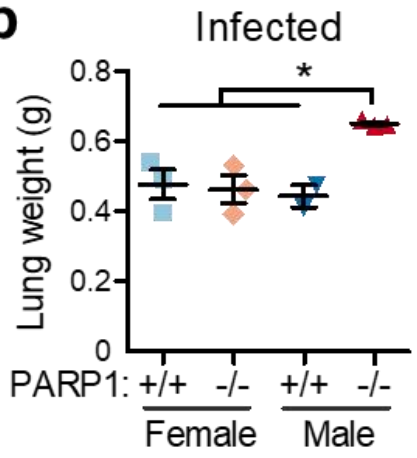

Day 28

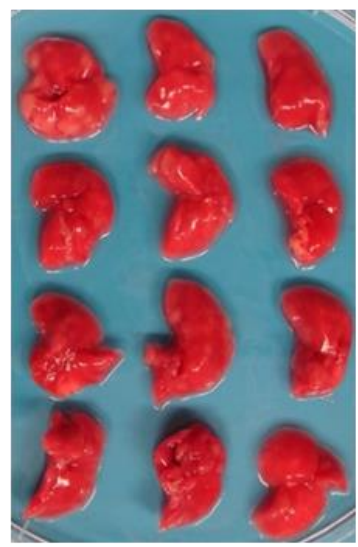

M

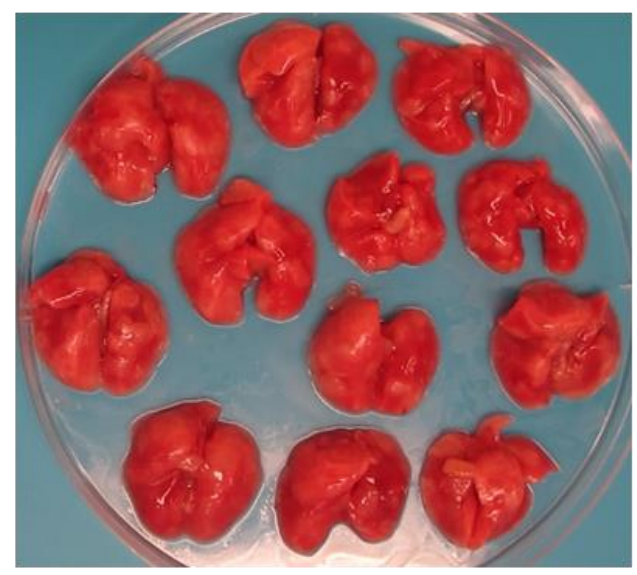

Day 74

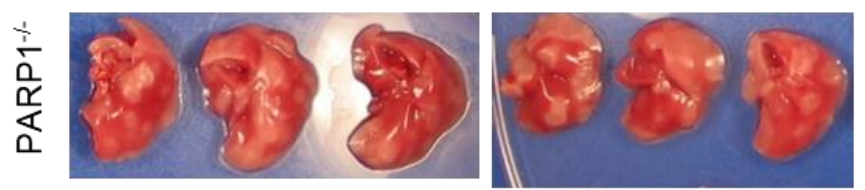

$1 \mathrm{~cm} \quad \lessgtr$

d

C

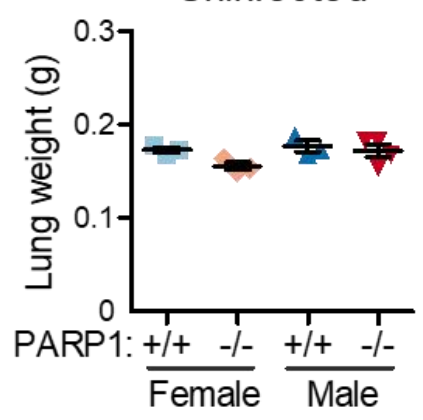

Fig. S4

Day 42
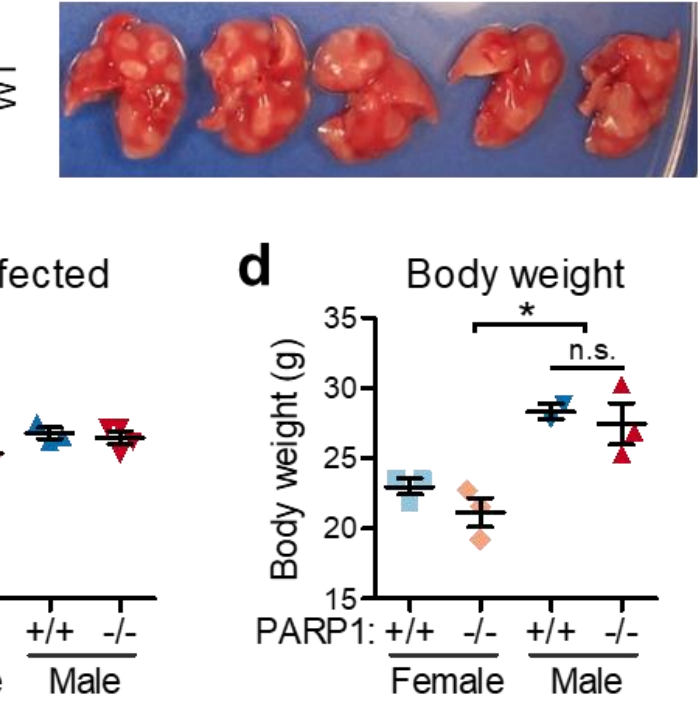

Figure S4. Chronically TB-infected male but not female PARP1-/- mice have enlarged lungs.

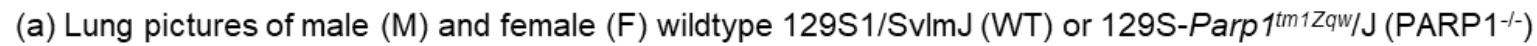
mice at 21, 28, 42, 56 and 74 days post aerosol infection with M.tb H37Rv. At days 28, 56 and 74, only the left lobes (pictured) were collected for gross pathology and CFU determination and the right lobes immediately processed for cytokine and PAR level analysis. (b-d) Average weight of lungs (b-c) or mice (d) 74 days post infection or of age-matched uninfected mice (c). ${ }^{*}, p<0.05 ;$ n.s., not significant by one-way ANOVA with Bonferroni post test. 


\section{Fig. S5}
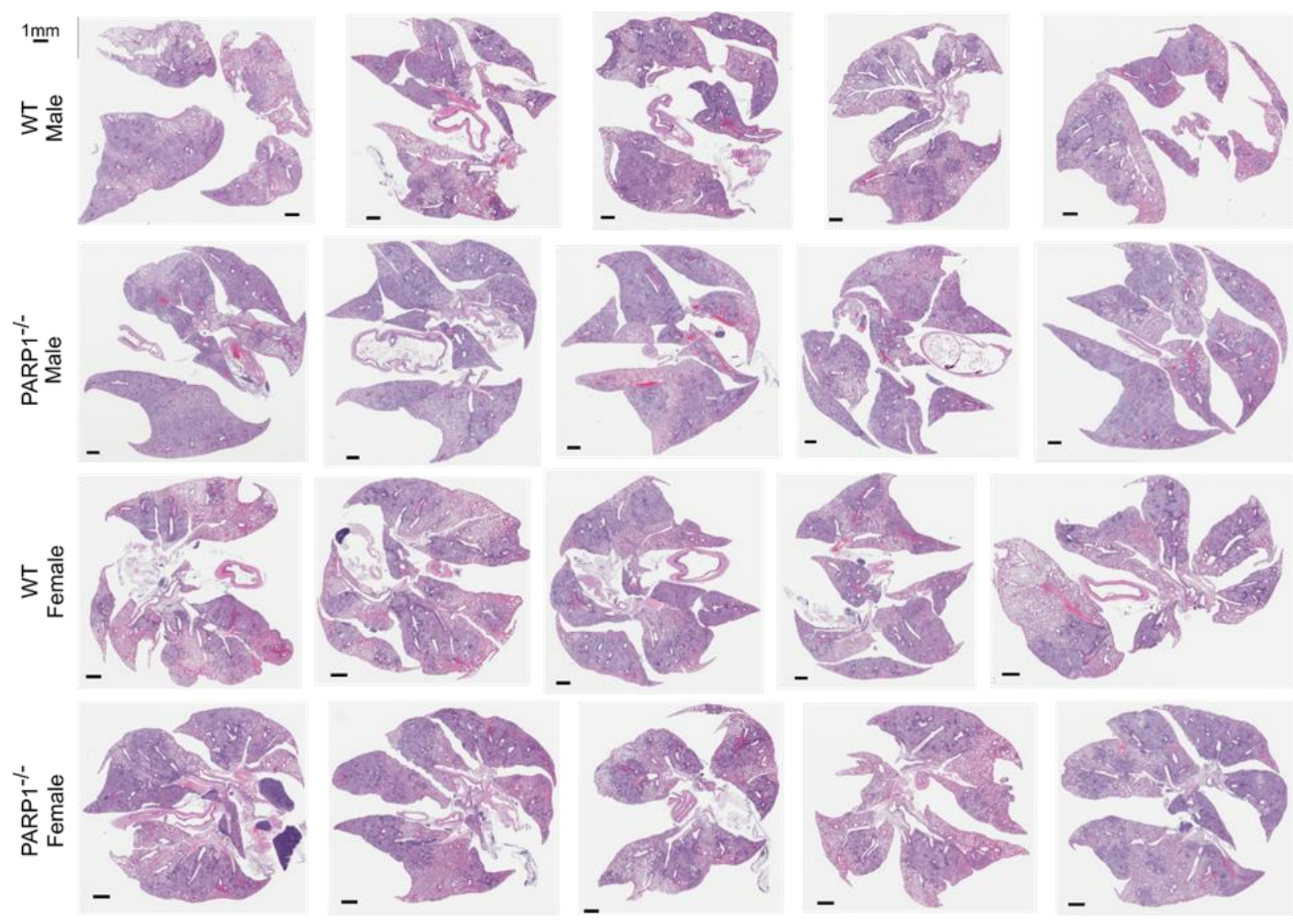

Figure S5. Histopathology reveals extensive lung consolidation in PARP1- males.

H\&E-stained lung sections of all male and female WT or PARP1-- mice 3 months after infection with M.tb H37Rv (day 1: $1.66 \log _{10} \mathrm{CFU}$ ). There was extensive consolidation throughout the lungs, in particular in PARP1--males. Quantification of consolidation and percent lung involvement indicating lung inflammation shown in figure 4. 


\section{Fig. S6}
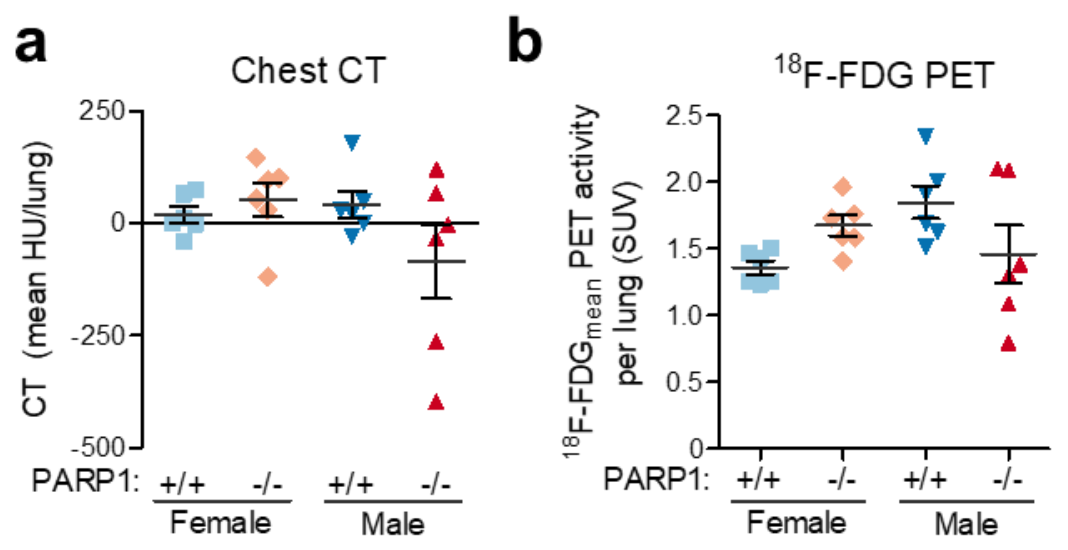

C

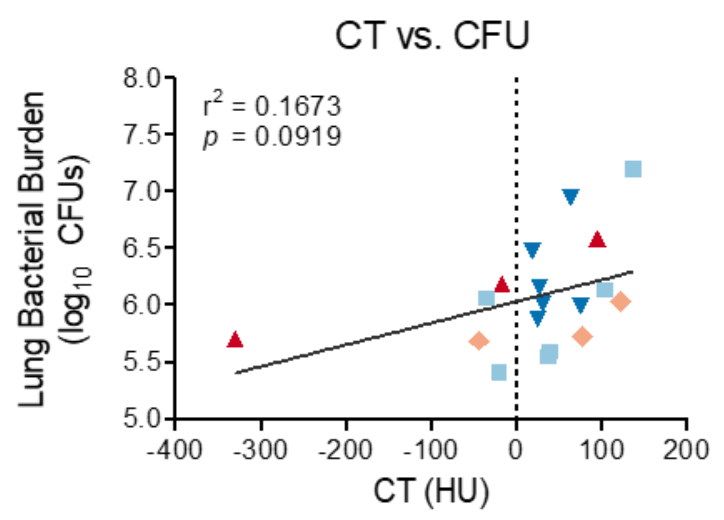

Figure S6. Inflammation analysis by ${ }^{18}$ F-FDG PET-CT.

Inflammation analysis in 3 male and 3 female WT or PARP1- mice 3 months after infection with M.tb H37Rv (day 1: $1.66 \log _{10}$ CFU) by CT (a) and ${ }^{18}$ F-FDG PET (b). Each data point represents the mean activity recorded in the left or the right lung ( 2 data points per mouse). (a) CT values of $-1000,0$ and $+1000 \mathrm{HU}$ indicate air, water and bone, respectively. CT values between $0-50 \mathrm{HU}$ indicate diseased lung. (b) Mean ${ }^{18}$ F-FDG PET activity per lung was normalized to the injected dose and body weight. SUV = Standardized uptake value. (c) For each mouse, lung bacterial burden was plotted against CT (average of left and right lung). CT values correlate with bacterial burden. 
Fig. S7

a

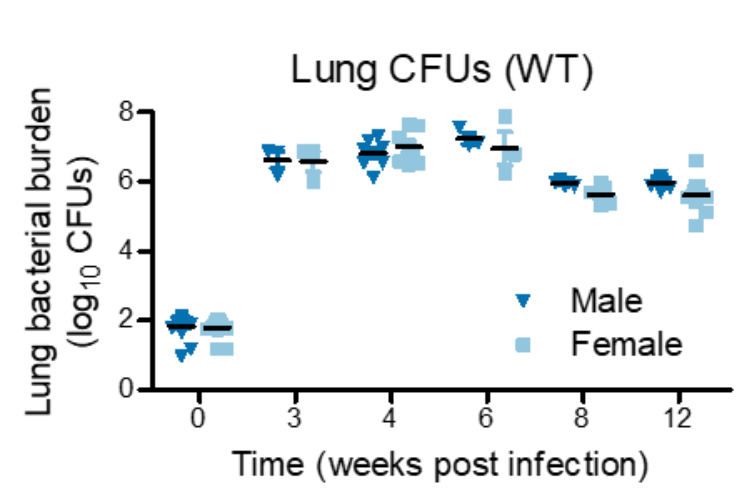

C

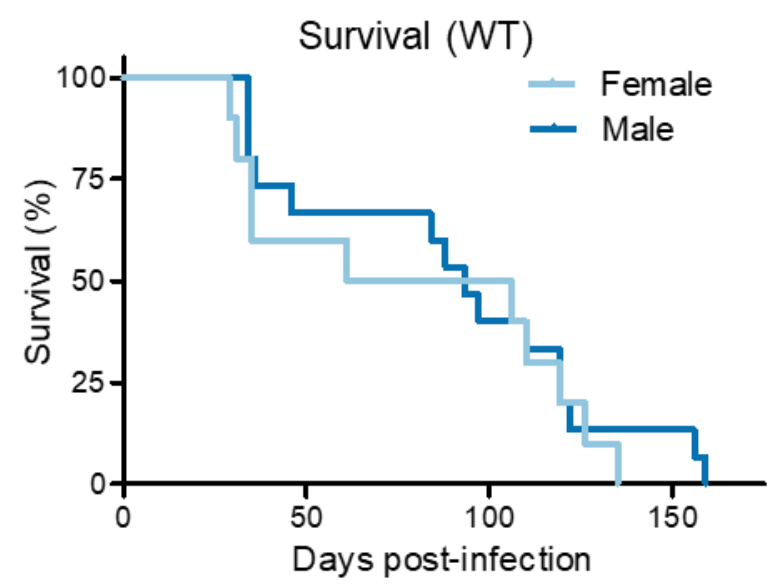

b

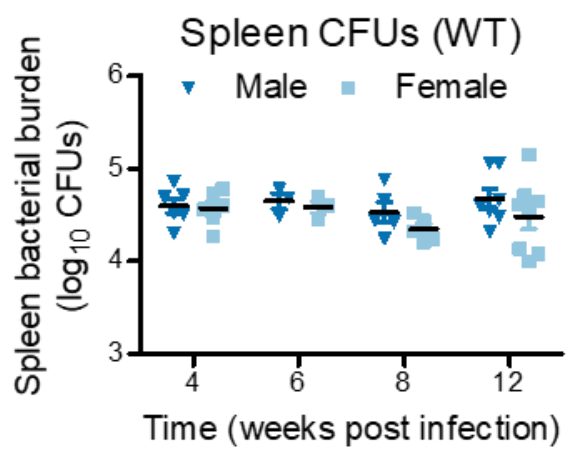

d

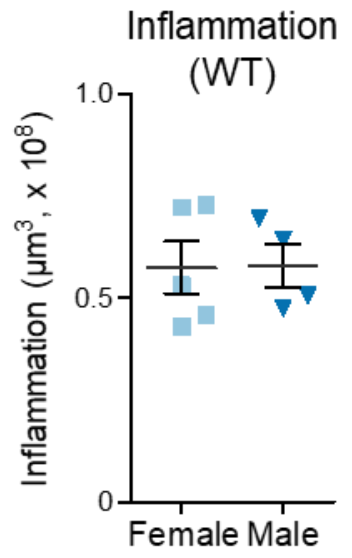

Figure S7. TB progression is comparable in male and female 129S1 WT mice.

(a-b) Time course of bacterial burden in the (a) lungs and (b) spleens of male and female 129S1/SvlmJ WT mice aerosol-infected with M.tb H37Rv or H37Rv $\Delta p n c A$. Data was collected in three independent experiments (day 1: $1.659 \pm 0.3172,1.724 \pm 0.0916$ and $1.858 \pm 0.2356$ $\log _{10} \mathrm{CFU}( \pm \mathrm{SD})$ ). Each data point represents an individual mouse. Lines indicate mean and SEM. (c) Survival in male and female 129S1/SvImJ WT mice $(n=15)$ following aerosol infection with M.tb H37Rv (day 1: $2.397 \log _{10} \mathrm{CFU}$ ). (d) Lung inflammation in male and female 129S1/SvIm WT mice 3 months after aerosol infection with M.tb H37Rv (day 1: $1.66 \log _{10}$ $\mathrm{CFU}$ ). Each point represents the area of consolidation, determined histologically, in an individual mouse. There were no apparent sex differences in bacterial burden, survival or lung inflammation in TB-infected WT mice. 


\section{Fig. S8}

a

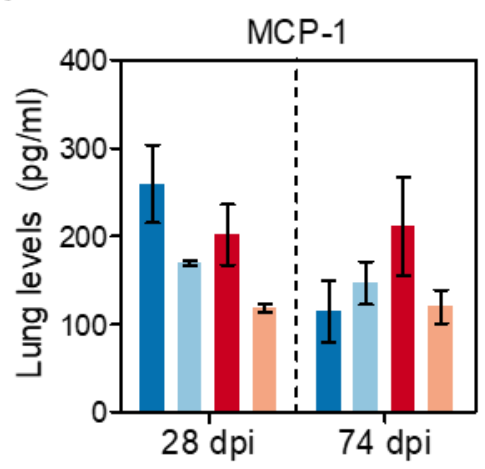

C

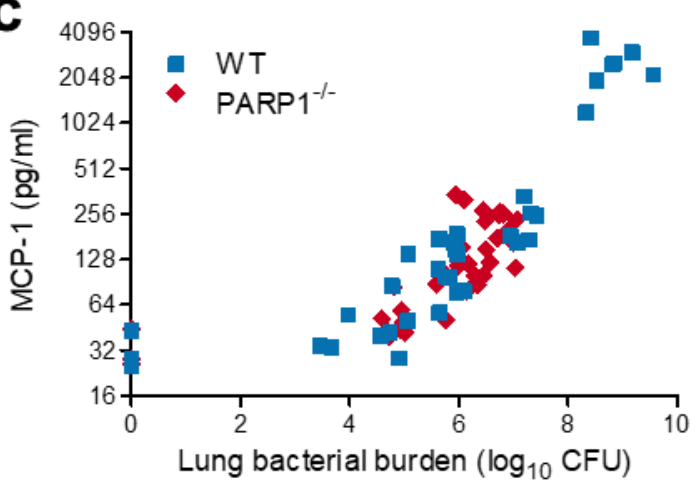

b

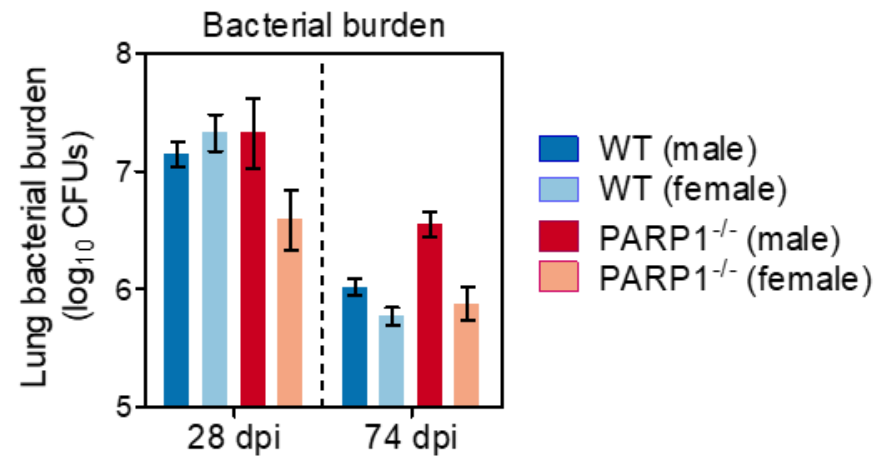

Correlation analysis: MCP-1 vs. CFU

WT

PARP1 $\%$

$r$

0.9227

0.7975

$\mathrm{Cl} \quad 0.851-0.961$

$0.638-0.891$

$p \quad<0.0001 \quad<0.0001$

Figure S8. MCP-1 correlates with bacterial burden in WT and PARP1-- mice.

(a-b) MCP-1 levels (a) and bacterial burden (b) in the lungs of male and female WT (blue) and PARP1-/ (maroon) mice 28 ad 74 days after aerosol infection with M.tb H37Rv (implantation: $1.86 \log _{10} \mathrm{CFU}$ ). Lung cytokines were quantified by Luminex multiplex assay. At $28 \mathrm{dpi}$, PARP1\%-females have the lowest MCP-1 levels and lowest bacterial burden, and at $74 \mathrm{dpi}$, PARP1\%-males have the highest MCP-1 levels and highest bacterial burden. (c) Spearman correlation analysis of bacterial burden vs. MCP-1 levels in WT (blue) and PARP1 $1_{-1}^{-}$(red) mice. Each point represents an individual mouse. $r$, correlation coefficient. $\mathrm{Cl}, 95 \%$ confidence interval. MCP-1 levels correlate with bacterial burden in WT and PARP $1^{-/-}$mice. 